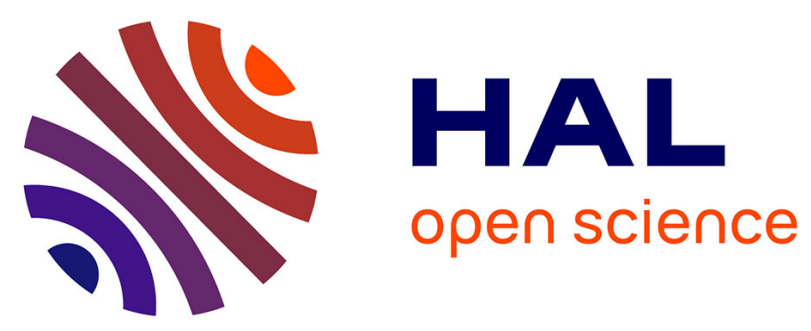

\title{
Generation of Interconnected Neural Clusters in Multiscale Scaffolds from Human-Induced Pluripotent Stem Cells
}

Boxin Huang, Juan Peng, Xiaochen Huang, Feng Liang, Li Wang, Jian Shi, Ayako Yamada, Yong Chen, Juan Peng-Wang

\section{To cite this version:}

Boxin Huang, Juan Peng, Xiaochen Huang, Feng Liang, Li Wang, et al.. Generation of Interconnected Neural Clusters in Multiscale Scaffolds from Human-Induced Pluripotent Stem Cells. ACS Applied Materials \& Interfaces, 2021, 13 (47), pp.55939-55952. 10.1021/acsami.1c18465 . hal-03442115

\section{HAL Id: hal-03442115 https://hal.science/hal-03442115}

Submitted on 26 Nov 2021

HAL is a multi-disciplinary open access archive for the deposit and dissemination of scientific research documents, whether they are published or not. The documents may come from teaching and research institutions in France or abroad, or from public or private research centers.
L'archive ouverte pluridisciplinaire HAL, est destinée au dépôt et à la diffusion de documents scientifiques de niveau recherche, publiés ou non, émanant des établissements d'enseignement et de recherche français ou étrangers, des laboratoires publics ou privés. 


\section{Generation of interconnected neural clusters in}

\section{multiscale scaffolds from human induced pluripotent}

stem cells

Boxin Huang ${ }^{1}$, Juan Peng-Wang ${ }^{1}$, Xiaochen Huang ${ }^{1}$, Feng Liang ${ }^{1}$, Li Wang ${ }^{2}$, Jian Shi ${ }^{2}$, Ayako Yamada ${ }^{1 *}$, Yong Chen ${ }^{1 *}$

${ }^{1}$ PASTEUR, Département de chimie, École normale supérieure, PSL Université, Sorbonne Université, CNRS, 75005 Paris, France

${ }^{2}$ MesoBioTech, 231 Rue Saint-Honoré, 75001, Paris, France

KEYWORDS: hiPSC, multiscale scaffolds, nanofibers, neural clusters 


\section{ABSTRACT}

The development of in vitro neural networks depends to a large extent on the scaffold properties, including the scaffold stiffness, porosity, and dimensionality. Herein, we developed a method to generate interconnected neural clusters in a multiscale scaffold consisting of a honeycomb microframe covered on both sides with a monolayer of crosslinked gelatin nanofibers. Cortical neural precursor cells (NPCs) were firstly produced from human induced pluripotent stem cells (hiPSCs) and then loaded into the scaffold for a long period of differentiation toward cortical neural cells. As result, neurons and astrocytes self-organized in the scaffold to form clusters in each of the honeycomb compartments with remarkable inter-cluster connections. These cells highly expressed neuron- and astrocyte-specific proteins, including NF200, Tau, Synapsin I and GFAP, and showed spatially correlated neural activities. Two types of neural clusters, i.e., spheroid-like and hourglass-like clusters, were found, indicating the complexity of neural-scaffold interaction and the variability of three-dimensional neural organization. Furthermore, we incorporated a reconstituted basement membrane into the scaffold and performed coculture of the neural network with brain microvascular endothelial cells. As a proof of concept, an improved neurovascular unit model was tested showing large astrocytic endfeet on the backside of the endothelium.

\section{INTRODUCTION}

The neocortex of the human brain has been developed for high-order cognitive and mental activities, which involves a huge number of neurons and glial cells ${ }^{1-2}$. These cells are organized orderly and powered by glucose and oxygen from brain blood vessels ${ }^{3}$. Although the neocortex is highly complex, its building block, neurovascular unit (NVU), is conceptionally simpler, 
which is composed of a limited number of neurons and glial cells, pericytes, microvascular endothelial cells, as well as brain extracellular matrix proteins ${ }^{4}$. In a NVU, neurons and glial cells form three-dimensional (3D) neural networks and other cells line on a basement membrane (BM) to form a blood-brain barrier (BBB) which is selectively permeable to small molecules but highly protective to drugs ${ }^{5}$. Of note, the integrity of the NVU is vital to the hemostasis and activity of the brain, since many neurodegenerative diseases, including Alzheimer's and Parkinson diseases, are due to damage or disorder of the $\mathrm{NVU}^{6}$ or BBB $^{7}$. In this regard, in vitro modeling of the 3D neural network, the BBB, and the NVU is all-important for fundamental research and brain therapy ${ }^{8-11}$.

Previously, a large number of in vitro studies has been devoted to the neural networks and BBBs ${ }^{12-16}$ but only a few of them focused on NVU modeling ${ }^{17-19}$. Moreover, many of the previous studies relied on animal cells or immortalized cell lines, which are inappropriate for modeling neither neocortical neural networks $(\mathrm{CNN})$ nor $\mathrm{BBB}$, because of significant differences in species or lack of multicellular types ${ }^{20}$. Human-induced pluripotent stem cells (hiPSCs) are now widely used since they are originated from human cells, self-renewable, and differentiated to different adult cells ${ }^{21}$. Excitedly, hiPSCs can also be used to generate brain organoids ${ }^{22-23}$, which are advantageous for brain developmental studies and simple drug assays but limited to suspension culture. Blood vessel organoids can also be derived from hiPSCs ${ }^{24}$. Although the brain organoids can be cultured for a long time and two organoids can be fused ${ }^{25}$, they are generally small, immature, and lack of immune-cell mediated competence. Nevertheless, organoids are inherently multicellular systems containing several types of correlated cells and can be disassociated into single cells before replating on a flat or patterned substrate ${ }^{26}$. Since there hiPSCs derived cells are subjected to a new environment, a period of adaptation, network 
organization, and neural network maturation is needed. Lineage-specific differentiation of hiPSCs allows producing different types of neural cells independently, including neurons and astrocytes ${ }^{27}$. Similarly, the coculture of these cells is possible but the development and optimization of a designed neural system seems to be time-consuming and laborious ${ }^{27,28}$. Finally, directed differentiation of hiPSCs allows creating at once neural networks with multicellular components, which simpler and more robust in terms of electrophysiologically mature network formation ${ }^{29}$. Depending on the early commitment and the differentiation protocol, different types of networks, including CNNs, should be achievable. We, therefore, adopted this latter strategy by firstly generating cortical committed neural precursor cells (NPCs) and then differentiating them with a patterned bilayer nanofiber scaffold toward 3D-CNNs and NVUs, with or without incorporation of an artificial BM.

We are particularly interested in neural networks made up of self-organized and interconnected neural clusters, which can be used to investigate multiscale and hierarchic neural systems ${ }^{30,31}$. Previously, most of the proposed 3D scaffolds for neural culture consisted of randomly distributed pores ${ }^{32,33}$ or gel structures ${ }^{34,35}$, which are inflexible for a controlled network organization. To overcome this drawback, ordered colloidal substrates, patterned or 3D scaffolds were developed to facilitate the growth and analysis of hiPSC-derived neurons ${ }^{13,36,37}$. In addition, a large variety of microfluidic devices have been proposed to control the outgrowth of neurons or to model neurogenerative disorders ${ }^{38,39}$, but few of them focused on CNN or NVU modeling. We wish to evaluate the feasibility of in-vitro NVU modeling by cocultivation of 3D neural networks with endothelial cells. In this regard, a scaffold containing artificial BM is desirable. Transwell-like devices are widely used in BBB studies, where endothelial cells are placed on the upside and neural cells in the downside of a semipermeable plastic membrane ${ }^{40}$. 
This device configuration is simple and convenient for culture and monitoring but is oversimplified since there are significant differences between a plastic membrane and a natural BM in terms of stiffness, porosity, and bioreactivity ${ }^{41}$. Herein, we present a method to firstly create 3D CNNs from hiPSC in compartmented bilayer scaffold consisting of two monolayers of crosslinked gelatin nanofibers and a lithography-defined honeycomb microframe. Then, we transform one of the nanofiber monolayers to artificial BM to coculture the CNNs with endothelial cells. Phase-contract images and immunofluorescence images are shown to elucidate the formation of self-organized 3D neural clusters in the scaffold as well as the feasibility of in vitro modeling of $\mathrm{BBB}$ and $\mathrm{NVU} . \mathrm{Ca}^{2+}$ transient images are analyzed to illustrate the intralayer and interlayer correlation of neural activities.

\section{EXPERIMENTAL SECTION}

Reagents were purchased from Sigma-Aldrich unless otherwise specified.

\subsection{Fabrication of the scaffold}

Our scaffold was made of crosslinked gelatin nanofibers on both sides of a $150 \mu \mathrm{m}$-thick plastic frame with pre-defined hexagonal through holes. The scaffold is supported with a plastic ring of $9 \mathrm{~mm}$ inner diameter and $13 \mathrm{~mm}$ outer diameter and $150 \mu \mathrm{m}$ thickness, providing a seaffold work area of $9 \mathrm{~mm}$ diameter in size. The side-to-side distance of hexagonal holes is 200 $\mu \mathrm{m}$ and the frame width is $50 \mu \mathrm{m}$, giving rise to 800 compartments in a scaffold. The frame structure was firstly produced by photolithography and then replicated in PDMS by casting and finally in a photoresist by vacuum-assisted UV-molding. Briefly, the frame pattern was defined in a chromium photomask (Microresist) by a micro-pattern generator ( $\mu$ PG 101, Heidelberg Instruments). The pattern was then replicated into a $150 \mu \mathrm{m}$ thick resist layer (SU-8 3050, 
MicroChem) spin-coated on a silicon wafer (Neyco). Afterward, the ring pattern was added by spin-coating another $150 \mu \mathrm{m}$-thick SU8 resist layer on the patterned layer. The SU-8 resist layers were prebaked prior to UV exposure and post-exposure bake. After the second exposure, the layers were developed in SU-8 developer (MicroChem) and rinsed with isopropanol. After being exposed to a vapor of chlorotrimethylsilane, the SU8 resist pattern was used as a template for PDMS casting. PDMS polymer and crosslinker (RTV 615, Momentive Performance Materials) were mixed at a ratio of 10:1 (w/w), poured on the SU-8 mold, degassed, and allowed to reticulate at $75^{\circ} \mathrm{C}$ for $4 \mathrm{~h}$. After being peeled off, inlet and outlet holes were punched through the PDMS block. The PDMS block was then placed on a glass slide with the structured-side down and kept under vacuum for 10 min immediately before injection of the OrmoStamp(Micro Resist Technology), a low viscosity UV sensitive prepolymer, into the cavity between the PDMS structure and the glass slide. Once OrmoStamp fulfilled the cavity, the assembly was exposed to UV light for $2 \mathrm{~min}$ at $21.6 \mathrm{~mW} \cdot \mathrm{cm}^{-2}$ and then removed from the glass slide. Finally, the scaffold made in OrmoStamp was peeled off from the mold.

For electrospinning deposition of nanofibers, a $10 \mathrm{~nm}$-thick gold layer was deposited on both sides of the frame structure with a sputter (K6785X, Emitech). Gelatin nanofibers were electrospun on the frame as described previously ${ }^{42-44}$. Gelatin from porcine skin was dissolved at $15 \mathrm{wt} \%$ in a solvent mixture containing distilled water, ethyl acetate, and acetic acid at a volume ratio of 10:14:21 and used in 2 days. The gelatin solution was ejected at a flow velocity of 0.2 $\mathrm{mL} \cdot \mathrm{h}^{-1}$ by using a syringe pump (Harvard Apparatus) with a syringe needle at a distance of 10 $\mathrm{cm}$ under a bias voltage of $11 \mathrm{kV}$. Gelatin nanofibers were electrospun for 2 min on both sides of the frame and cut off at their edges.

\subsection{Incorporation of ABM into the scaffold}


The electrospinning time was increased to $5 \mathrm{~min}$ to deposit more nanofibers on the bottom side of the frame. After removing the residual solvent in a desiccator, the gelatin nanofibers were crosslinked by incubation in $0.2 \mathrm{M} \mathrm{N}$-(3-Dimethylaminopropyl)-N'-ethylcarbodiimide hydrochloride and $0.2 \mathrm{M} \mathrm{N}$-hydroxysuccinimide in ethanol for $4 \mathrm{~h}$ at room temperature. The scaffold was rinsed in ethanol three times, dried under vacuum, and stored in a cool and dry place. Afterward, one of the nanofiber layers was used as backbone for the formation of ABM. The scaffold was firstly sterilized in a $70 \%$ ethanol solution and under UV light. Then, a $50 \mu \mathrm{L}$ solution of laminin $\left(20 \mu \mathrm{g} \cdot \mathrm{mL}^{-1}\right.$, Sigma-Aldrich $)$ and type IV collagen $\left(1 \mathrm{mg} \cdot \mathrm{mL}^{-1}\right.$, SigmaAldrich) in DI water was dropped on. After dehydration in an incubator at $37^{\circ} \mathrm{C}$ for more than 4 $\mathrm{h}$, the deposited ECM proteins were self-assembled in the porous areas of the nanofibers to form an ultrathin membrane.

\section{3 hiPSCs differentiation into NPCs}

hiPSCs (episomal line, Life Technologies) were cultured in Essential 8 Flex Medium (E8FM, Thermo Fisher Scientific) at $37{ }^{\circ} \mathrm{C}$ and $5 \% \mathrm{CO}_{2}$ on a tissue culture dish, which was treated with $5 \mu \mathrm{g} \cdot \mathrm{mL}^{-1}$ vitronectin (VTN-N, Thermo Fisher Scientific) in PBS for $1 \mathrm{~h}$ at $37{ }^{\circ} \mathrm{C}$ prior to use. The hiPSCs were subsequently differentiated into NPCs by following the protocol described with a slight modification ${ }^{29}$. First, dissociated hiPSCs were seeded on an ultra-lowattachment round-bottom 96 well plates (Corning) at a cell density of 5000-10,000 per well, and allowed to form embryoid bodies (EBs) in E8FM for 2 days. The culture medium was then switched to neural induction medium (NIM) composed of DMEM/F12 medium, $1 \% \mathrm{~N}_{2}$ supplement (Thermo Fisher Scientific), $2 \mu \mathrm{g} \cdot \mathrm{mL}^{-1}$ heparin (Stemcell), and $1 \%$ penicillinstreptomycin (Pen-strep, Thermo Fisher Scientific), in which cells were cultured for 4 days. On day 7 , EBs were transferred on a tissue culture plate, which was incubated with $20 \mu \mathrm{g} \cdot \mathrm{mL}^{-1}$ 
laminin in PBS for $1 \mathrm{~h}$ at $37^{\circ} \mathrm{C}$ beforehand, and cultured in NIM until day 14 to generate preNPCs. Pre-NPCs were then cultured in neural precursor medium (NPM) composed of DMEM/F12 medium, $1 \% \mathrm{~N}_{2}$ supplement, $2 \% \mathrm{~B} 27$ minus vitamin A supplement (Thermo Fisher Scientific), $1 \mu \mathrm{g} \cdot \mathrm{mL}^{-1}$ laminin, $20 \mathrm{ng} \cdot \mathrm{mL}^{-1}$ basic fibroblast growth factor (Stemcell), and $1 \%$ Pen-strep from day 15 onward. The medium was renewed every 2 days and cells were subcultured on a dish treated beforehand with $1 \%$ Geltrex (LDEV-free, reduced growth factor, Thermo Fisher Scientific) in DMEM/F12 medium for $1 \mathrm{~h}$ at $37^{\circ} \mathrm{C}$ when confluent. After 5 passages, cells were considered as NPCs.

\subsection{NPC differentiation and neural cluster formation}

Prior to cell seeding, both sides of a bilayer scaffold were treated by air plasma (Harrick Plasma) for 1 min each, and the scaffold was sterilized by UV irradiation in $70 \%$ ethanol for 30 min. After being rinsed with PBS and dried at room temperature, the bilayer scaffold was incubated with $100 \mu \mathrm{g} \cdot \mathrm{mL}^{-1}$ poly-L-ornithine at $4{ }^{\circ} \mathrm{C}$ overnight and rinsed 3 times with PBS. A droplet of $1 \%$ Geltrex in DMEM/F12 medium was deposited on the scaffold and incubated for 1 $\mathrm{h}$ at $37^{\circ} \mathrm{C}$ in a humidified incubator. Next, NPCs were detached using Accutase (Thermo Fisher Scientific), seeded on the flat "bottom" side of bilayer scaffold at a density of $3.0 \times 10^{5}$ cells per scaffold, incubated for $2 \mathrm{~h}$ at $37{ }^{\circ} \mathrm{C}$ and $5 \% \mathrm{CO}_{2}$ for cell adhesion, and kept cultured in $1 \mathrm{~mL}$ NPM. The next day, $3.0 \times 10^{5}$ NPCs were seeded in the same manner on the "top" side of the scaffold flipped beforehand, and cells were further differentiated in neural differentiation medium (NDM) composed of Neurobasal medium (Thermo Fisher Scientific) supplemented with $1 \% \mathrm{~N}_{2}$ supplement, $2 \% \mathrm{~B} 27$ minus vitamin A supplement, $20 \mathrm{ng} \cdot \mathrm{mL}^{-1}$ brain-derived

neurotrophic factor (Stemcell), $20 \mathrm{ng} \cdot \mathrm{mL}^{-1}$ glial cell-derived neurotrophic factor (Stemcell), $1 \%$ Pen-strep, $1 \%$ non-essential amino acid solution (Thermofisher), $1 \mu \mathrm{M}$ dibutyryl cyclic 
adenosine monophosphate and $2 \mu \mathrm{g} \cdot \mathrm{mL}^{-1}$ laminin. NDM was fully renewed every two days for 4 weeks and half the volume was renewed onwards.

\subsection{Creation of neurovascular unit}

The bilayer scaffold incorporated with an ABM was used for the creation of the NVU. To promote cell adhesion, the scaffold was also coated by poly-L-ornithine and Geltrex. The cell processing protocol is displayed in Figure 7B. Firstly, $3.0 \times 10^{5}$ NPCs were seeded on the top side of the gelatin nanofibers in NPM and differentiated into neural networks in NDM for 8 weeks. The NDM medium was replaced by that for neural clusters formation. Subsequently, 4.0

$\times 10^{5}$ brain microvascular endothelial cells (hCMEC/D3 cells) were seeded at the bottom side of the ABM in endothelial cells growth medium (EGM), while the on-scaffold neural networks were still immersed in NDM. The bilayer scaffold was mounted into a device with PDMS contact layers and upper and lower chambers for incubation with different types of culture medium. After $2 \mathrm{~h}$ incubation at $37{ }^{\circ} \mathrm{C}$ and $5 \% \mathrm{CO}_{2}$ for cell adhesion, the bilayer scaffold was transferred into a mixed medium (NDM: EGM=1:1) for further coculture. The mixed medium was changed every two days.

\subsection{SEM observation}

A $5 \mathrm{~nm}$-thick gold layer was sputtered on the bilayer scaffold prior to observations by SEM (Hitachi TM3030) operated at $10 \mathrm{kV}$. The scaffold was cut by a blade to obtain a cross-sectional view.

\subsection{Immunofluorescence staining}


Cells were fixed by incubation in $4 \%(\mathrm{w} / \mathrm{v})$ paraformaldehyde in PBS for $15 \mathrm{~min}$, prior to permeabilization in $0.5 \%(\mathrm{w} / \mathrm{v})$ Triton X-100 in PBS for $10 \mathrm{~min}$ and incubation in a blocking solution ( $3 \%$ bovine serum albumin, $0.1 \%$ Tween 20 , and $0.1 \%$ sodium azide $(w / v)$ in PBS) for $30 \mathrm{~min}$ at room temperature. The samples were further incubated with primary antibodies at $4{ }^{\circ} \mathrm{C}$ overnight, followed by the incubation with secondary antibodies in the blocking solution for $2 \mathrm{~h}$ and subsequent nuclear staining in PBS containing $1 \mu \mathrm{g} \cdot \mathrm{mL}^{-1}$ DAPI for $15 \mathrm{~min}$ at room temperature. Besides, the F-actin staining was made by incubating cells in ActinRed 555 ReadyProbes (Thermo Fisher Scientific, 1: 20) for $30 \mathrm{~min}$ at room temperature before cell nuclear staining. The following primary antibodies were used for immunostaining: anti-Nestin (N5413, Sigma-Aldrich, 1:100 dilution), anti-BIII tubulin (T8578, Sigma-Aldrich, 1:500), antiGFAP (MA5-12023, Thermo Fisher Scientific, 1:100), anti-Neurofilament 200 (N4142, SigmaAldrich, 1:80), anti-Tau (T9450, Sigma-Aldrich, 1:100), anti-Synapsin I (ab64581, abcam, 1:200). Alexa fluor 488 anti-mouse (A-21200, 1:500), Alexa fluor 488 anti-rabbit (A32790, 1:500), Alexa fluor 633 anti-mouse (A-21050, 1:500), and Alexa fluor 633 anti-rabbit (A-21070, 1:500) antibodies (Thermo Fisher Scientific) were used as secondary antibodies. Fluorescently labeled cells were imaged by confocal microscopy (LSM 900, Zeiss).

\subsection{Calcium imaging and analysis}

Cells were rinsed with a recording buffer $\left(145 \mathrm{mM} \mathrm{NaCl}, 3 \mathrm{mM} \mathrm{KCl}, 1.5 \mathrm{mM} \mathrm{CaCl}_{2}, 1 \mathrm{mM}\right.$ $\mathrm{MgCl}_{2}, 10 \mathrm{mM}$ glucose, and $10 \mathrm{mM}$ HEPES, $\mathrm{pH}$ 7.4) and loaded with $2 \mu \mathrm{M}$ Fluo-4 AM and $20 \%$ pluronic acid (both from Thermo Fisher Scientific) in the recording buffer at $37{ }^{\circ} \mathrm{C}$ in a $5 \% \mathrm{CO}_{2}$ incubator for $25 \mathrm{~min}$. After incubation, the samples were rinsed with the recording buffer twice and transferred to the stage of an inverted fluorescence microscope (Axio Observer 
Z1, Zeiss) equipped with an Electron Multiplier CCD Camera C11440 (Hamamatsu Photonics).

The experiment was performed at room temperature and each frame was acquired every $500 \mathrm{~ms}$ for 5-10 minutes. Regions of interest (ROIs) covering single cell bodies or cell clusters were selected in a semi-automated manner in z-stack images and the fluorescence intensity in each ROI in each frame was measured by Image J, which allowed us to identify the location of cell bodies or cell clusters and to analyze $\mathrm{Ca}^{2+}$ transient dynamics of the cell population in 3D space. The normalized fluorescence signal was calculated as follows: $\Delta \mathrm{F} / \mathrm{F}=(\operatorname{If}(\mathrm{t})-\operatorname{If}(\mathrm{t} 0)) / \operatorname{If}(\mathrm{t} 0)$, where $\operatorname{If}(\mathrm{t} 0)$ is the average fluorescence intensity at the beginning of the recording and If $(\mathrm{t})$ is the time course of the average fluorescence intensity in an ROI. Further, an interactive Fluorescence Single Neuron and Network Analysis Package (FluoroSNNAP) was used to gain deeper insights into the $\mathrm{Ca}^{2+}$ transients ${ }^{45}$. The package allowed semi-automated identification of ROIs and calculation of $\Delta \mathrm{F} / \mathrm{F}=\left(\mathrm{If}(\mathrm{t})-\mathrm{If}_{\text {-base }}\right) / \mathrm{If}_{\text {-base }}$ in each ROI, where $\mathrm{If}_{\text {-base }}$ is the mean of the lower $50 \%$ of previous 10 -s values. A network analysis module from the Package was used to investigate the synchrony and functional connectivity of the samples.

\subsection{Static analysis}

Data are shown as the standard error of the mean from at least three independent samples. P values were calculated by unpaired Student's t-test by the software GraphPad. The number of replicates and statistical tests used for each experiment are mentioned in the respective figure legends. Significance was set to $* \mathrm{P}<0.05,{ }^{* *} \mathrm{P}<0.01, * * * \mathrm{P}<0.01$.

\section{RESULTS}




\subsection{Fabrication of patterned bilayer nanofiber scaffolds}

The fabrication of the bilayer scaffolds was based on lithography and electrospinning techniques ${ }^{44}$. By varying the electrospinning time, the number of nanofibers deposited could be controlled. Ultimately, a monolayer of nanofibers could be obtained with desirable porosity ${ }^{42}$. As shown in Figures 1A, B, and S1A, a monolayer of crosslinked gelatin nanofibers was deposited on both sides of the honeycomb microframe. By analyzing the scanning electron microscopy images, an average nanofiber diameter of $\sim 500 \mathrm{~nm}$, a porosity of $\sim 20 \%$ and a monolayer thickness of $\sim 200 \mathrm{~nm}$ were determined. For such an ultrathin pore membrane, the diffusion flux is much higher than other types of membranes such as track-etched plastic membrane commonly used for cell culture or coculture, since it is reversely proportional to the thickness of the membrane. Statistically, $93 \%$ of the pores were smaller than $20 \mu \mathrm{m}^{2}$ and $55 \%$ of the pores were smaller than $5 \mu \mathrm{m}^{2}$ (Figure S1B). By using a similar technique as described ${ }^{\mathrm{xx}}$, an effective in-plan Young's modulus in the order of $10 \mathrm{kPa}$ was estimated. Gelatin is produced by partial hydrolysis of collagen so that it is inherently biocompatible. Our recent studies showed that electrospun gelatin nanofibers could be used as substrate for hiPSC propagation over many generations without any surface coating, proving the importance of nanofibrous surface morphology ${ }^{\mathrm{xx}}$. More recent studies showed that neural cells and in particular astrocytes on a surface coated monolayer of crosslinked gelatin nanofibers were more in-vivo like ${ }^{\mathrm{xx}}$. Due to its multiscale architecture, the monolayer nanofiber scaffold is also mechanically stable and can be easy handled for cell culture and device integration. Therefore, the bilayer nanofiber scaffold has unique advantages to combine ultrathin, high porosity, low stiffness, and high biocompatibility. Since the monolayer was also compartmented and the size, thickness and geometry of the compartment as well as the size and thickness of the nanofiber layer can be control, the bilayer 
scaffold enables cell filtration into the free space between the two nanofiber layers and it is therefore suited for guided 3D self-organization.

The as-fabricated scaffolds are ready to use for the 3D culture of NPCs. Furthermore, they can be transformed into more specific scaffolds by incorporating ABM that is requested for both BBB and NVU modeling. This has been done by dropping a solution of type IV collagen and laminin, the two major natural $\mathrm{BM}$ proteins, on one of the nanofiber monolayers, in a similar way of the fabrication with one monolayer of gelatin nanofibers ${ }^{46}$. During dehydration, the two types of BM proteins self-assembled in the nanofiber pores to form an $\mathrm{ABM}$ with the monolayer of crosslinked nanofibers as the backbone (Figure 1C and Figure 7A, right).

With or without $\mathrm{ABM}$, the bilayer scaffold is membrane like. By measuring the flowrate of a solution across the membrane as a function of applied pressure, a hydrodynamic resistance in the order of $1 \mathrm{mBar} . \mathrm{min} / \mathrm{mL}$ was deduced. Such a hydrodynamic resistance is porosity and thickness dependent and at least ten times smaller than that of track-etched membranes of $10 \mu \mathrm{m}$ thickness. In other words, the Darcy permeability of both types of membrane is much larger that of tracketched membranes, which is also in favor of culture or coculture under microfluidic conditions.

\subsection{Generation of three-dimensional and inter-connected neural clusters}

Neural precursor cells (NPCs) were derived from hiPSCs which can be expanded and cryopreserved by using the protocol described by Gunhanlar, et al. ${ }^{29}$ after slight modifications. As summarized in Figure 1D, hiPSCs were firstly cultured in a normal culture dish. After cell aggregation, hiPSC colonies were detached (Figure 1E, Day 0) and embryoid bodies (EBs) were formed in ultra-low attachment wells (Figure S2A, Day 2). After being cultured in a neural induction medium (NIM) for 3 days (Figure 1E, Day 5), EBs were transferred on a laminin- 
coated tissue culture plate on day 6 and cells started to spread on the substrate to form a homogeneous cell layer from day 6 to day 15 (Figure S2A, Day 7 and 14). The cells were then passaged at least 5 times to get NPCs in a neural precursor medium (NPM). As expected, the resulted NPCs showed characteristic expressions of neuronal progenitor marker Nestin, and neuron-specific cytoskeleton marker $\beta$-III tubulin (TUJ 1). As can be seen in Figure S2B, Nestin (red) was expressed in all the cells but TUJ 1 (green) was co-expressed in a part of the cell population, indicating that those cells were destined for neurons. Meanwhile, neural rosettes which are the signature of the neuronal progenitors were found.

Afterward, the dissociated NPCs were seeded on both sides of the scaffold (Figure 1E, Day 43) and cultured in a neural differentiation medium (NDM) (see "Method" for details). As the cells differentiated, many of them moved to the center and aggregated in each honeycomb compartment (Figure 1E, Day 100 and Figure S2C). Confocal microscopy images of F-actin and DAPI stained cells revealed that during the first week in the scaffold, the cells protruded not only on the nanofiber layers but also into the free space between the two nanofiber layers (Figure S2D, S2E). At this stage, although a majority of the cell bodies remained on the nanofiber layers, the number of cellular protrusions inside the scaffold increased with time (Figure 1F, Day 47). After differentiation in the scaffold for 8 weeks, more cells were self-organized in the center of each honeycomb compartment, forming spheroid-like cell clusters (neurospheroids) with radial extensions toward the edge of the compartment (Figure 1F, Day 100). These neural clusters could be stable over a long-period of incubation time (Figure 1E, Day 156).

The cell body distribution in the bilayer scaffold is shown in Figure 1G, which was obtained by $3 \mathrm{D}$ reconstitution of confocal z-stack images with DAPI staining. To better characterize the cell body distribution, we first divided radically the honeycomb compartment into 3 regions 
(Figure S3A) to calculate the number of nuclei in each of the regions. The central region A (radius $\mathrm{R} \leq 50 \mu \mathrm{m}$, pale yellow), the middle region $\mathrm{B}(\mathrm{R}=50 \sim 75 \mu \mathrm{m}$, yellow) and the outer region $\mathrm{C}(\mathrm{R}>75 \mu \mathrm{m}$, brown) contained $49.8 \pm 10.3 \%, 29.6 \pm 6.9 \%$, and $20.6 \pm 7.9 \%$ of nuclei, respectively (Figure 1G). This means that most of the cells were distributed in the center region of the honeycomb compartment. We then divided vertically the compartment also into 3 regions (Figure S3B) and calculated the average value of the radical distribution of the nuclei $r=67.4 \pm$ $11.2,35.1 \pm 19.6$, and $51.4 \pm 16.8 \mu \mathrm{m}$ in $\mathrm{H} 1(\mathrm{H}=0 \sim 50 \mu \mathrm{m}), \mathrm{H} 2(\mathrm{H}=50 \sim 100 \mu \mathrm{m})$, and $\mathrm{H} 3(\mathrm{H}$ $=100 \sim 150 \mu \mathrm{m})$, respectively. This means that the cells in the central region are mostly located in the middle layer $(\mathrm{H} 2)$ of the compartment (Figure $1 \mathrm{H})$. 
A

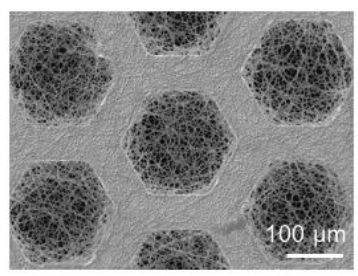

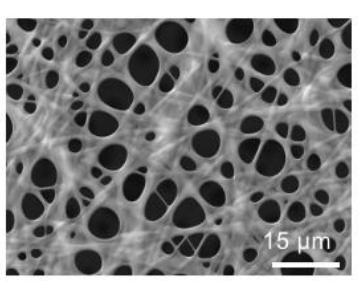
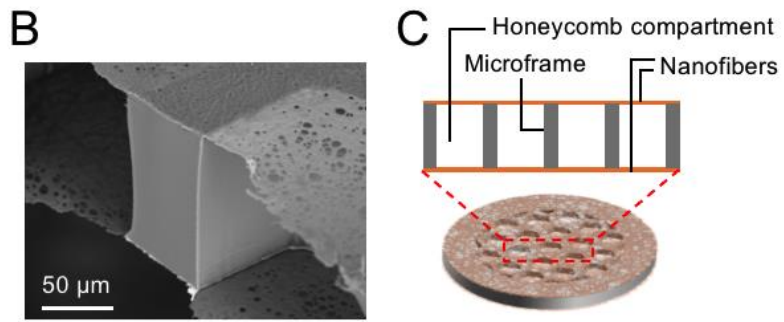

Bilayer nanofiber Patch

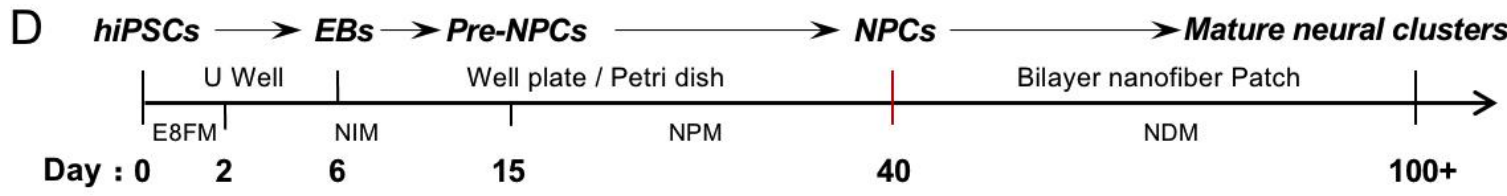

E
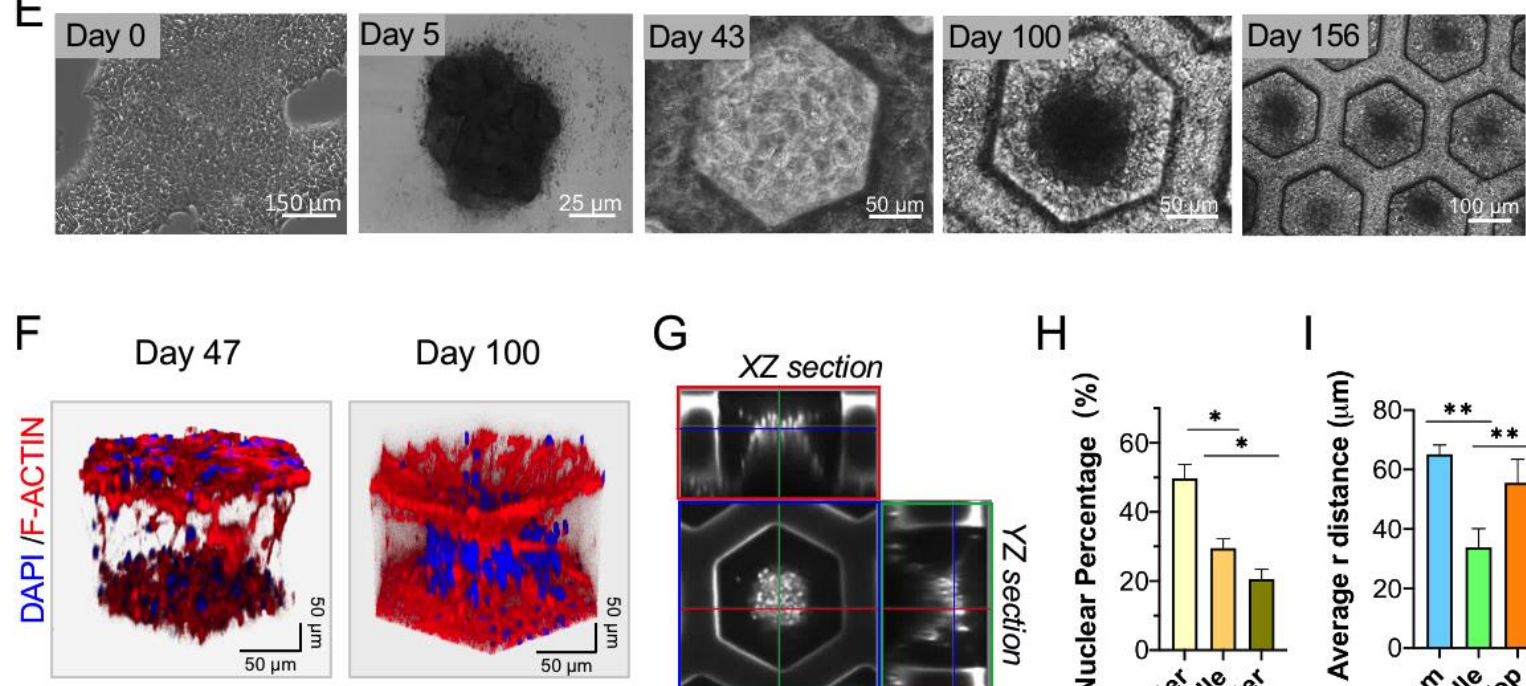

Day 100
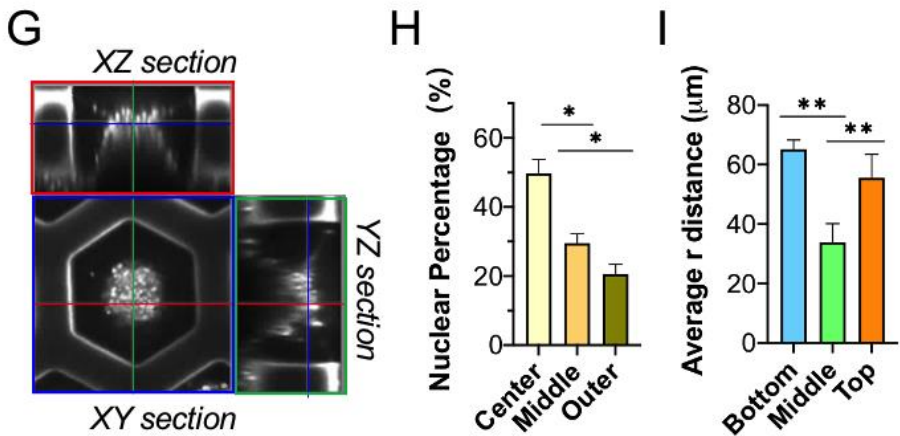

Figure 1. Development of interconnected neural clusters. A) Large-scale (left) and small-scale (right) SEM images of a scaffold made of crosslinked gelatin nanofibers onto a honeycomb microframe. B) Tilted-view SEM image of the scaffold showing two spaced nanofiber monolayers. C) Schematic diagram of the scaffold. D) Steps of hiPSC differentiation toward neuron precursor cells and cortical neural networks. E) Brightfield microscopy images of the cell organization during the whole period of neural network derivation. F) 3D-reconstructed images of confocal microscopy of F-actin (red) and nuclei (blue) of the cells at day 47 (left) and day 100 (right). G) Orthogonal views of the images of the cell nuclei inside a honeycomb compartment at day $100 . \mathrm{H}$ ) Distribution of the nuclei in the central (pale yellow), middle (yellow), and outermost (brown) regions inside a honeycomb compartment $(\mathrm{n}=3, \mathrm{P}<0.05$ student-t-test). I) Averaged radial distance of the cell nuclei (r) from the center in the bottom (H1, $\mathrm{H} \leqslant 50 \mu \mathrm{m}$, blue), middle ( $\mathrm{H} 2$, $50<\mathrm{H} \leqslant 100 \mu \mathrm{m}$, green) and top ( $\mathrm{H} 3,100<\mathrm{H} \leqslant 150 \mu \mathrm{m}$, orange) regions in a honeycomb compartment ( $\mathrm{n}=3, \mathrm{P}<0.01$ student-t-test). 
Neurons and astrocytes are the main cellular components of CNNs, in which astrocytes guide neural growth and support neuronal functions ${ }^{47,48}$. To evaluate the distribution of these cells, the neural networks formed in the bilayer scaffold after 8 weeks of differentiation (day 100) were stained by glial fibrillary acidic protein (GFAP) to detect astrocytes, neurofilament 200 (NF200) to detect neurons, in addition to the DAPI staining of the nuclei. Figure 2 shows Z projection (Figure 2A) and 3D views (Figure 2B, 2C) of confocal microscopy images of a typical area of the sample, revealing a self-organized network of neurons and astrocytes. Clearly, neural clusters were all localized in the center of honeycomb compartments and interconnected with dense and radial neurites and astrocytes. Moreover, the outgrowth of the neurites and astrocytes formed a bowl-like structure in each of the compartments. As marked by white arrows in the zoom image (Figure 2D), even single astrocytes are able to bridge two neighboring clusters (Figure 2E), indicating strong inter-cluster connections of the network. A more detailed observation suggested that some of the astrocytes were bundled and that most of the radial astrocytes were stretched (Figure 2D). In contrast, the astrocytes located on the frame area were guided by the surface of the frame but not stretched due to adhesion of the cells to the substate. In addition, a ring of astrocytic filaments could be observed in the center area of the honeycomb compartment, indicating a strong effect of self-organization. Accordingly, neurites followed closely the behavior of the astrocytes, showing also radial extension, geometric guiding, and rings (Figure 2A). Remarkably, the density of the neurites was significantly higher than that of the intermediate filaments in astrocytes, suggesting that the monolayer nanofiber scaffold is more favorable to the outgrowth of the neurites than that of the astrocytes. Generally speaking, the cell-material interactions affect differentiation of the cells and maturation of the neural networks ${ }^{49}$. In the present case, the outgrowth of neurites and astrocytes relied on monolayer of 
nanofibers which may result in less but more effective anchoring points in comparison with that on a flat surface. Furthermore, the suspended monolayer of nanofiber scaffold has a low effective stiffness but a high porosity (see below), which are both in favor of neural differentiation and neural network formation. As shown in Figure S3, in the early stage of neural differentiation, cells begun to aggerate in the nanofiber area and the outgrowth of neurites and astrocytic filaments were both affected by the morphology of nanofibers, except large size and stretched neural cells. With increase of the incubation time, more and more cells aggregated in the nanofiber areas and finally formed neural clusters. Finally, the cell-material interaction is less important and the effect of self-organization became dominant.

A
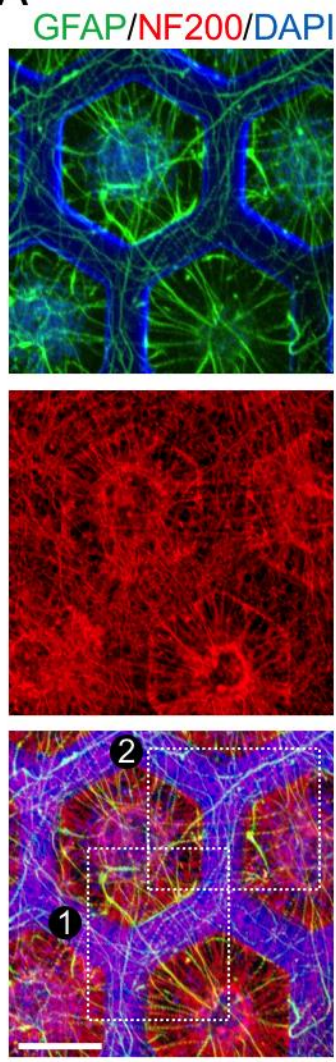

B

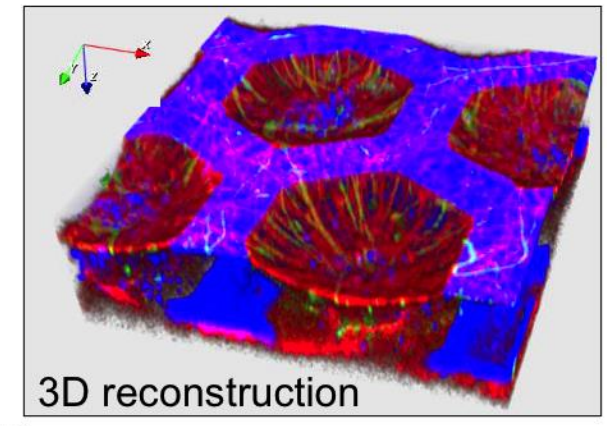

D

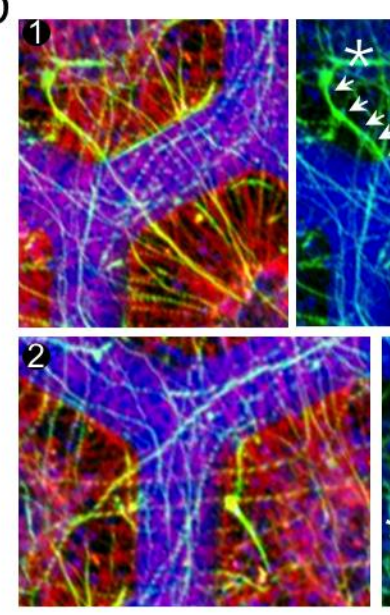

C

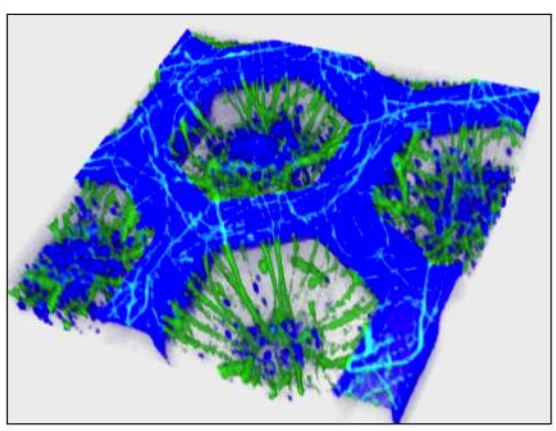

E

- Cell nuclei

Astrocyte

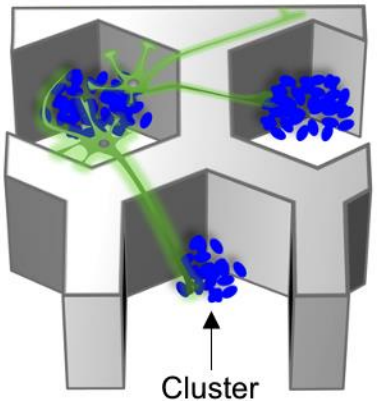


Figure 2. Confocal microscopy images of cortical neural networks. A) Cells were stained by DAPI (nuclei, blue), GFAP (astrocytes, green), and NF200 (neurons, red). Z-stack images were projected on an x-y plane. Scale bar, $100 \mu \mathrm{m}$. B) 3D reconstruction of the corresponding z-stack images with a height $150 \mu \mathrm{m}$. C) 3D reconstruction of DAPI and GFAP labeled z-stack images of heights between $100 \mu \mathrm{m}$ and $150 \mu \mathrm{m}$. D) Zoom-in of image A, indicating single astrocytes to bridge two neighboring clusters. The stars and arrow chains indicate the position of the neural clusters and the astrocytes, respectively. E) Artistic picture of the astrocyte connections cross two neighboring honeycomb compartments.

The distribution of neurons and astrocytes in a honeycomb compartment can be more clearly seen with 3D reconstruction and regional projection images (Figure 3). Overall, neurons and astrocytes were found on both sides and interlayer spaces of the scaffold. The spatial distribution and population of neurons and astrocytes on the scaffold are correlated (Figure 3B and Figure S4). While the interlayer neurons (NF200-stained) were mostly localized in the center region of the compartment, astrocytes (GFAP-stained) were more likely scattered around the neural cluster (Figure 3A). The regional z-projections of the confocal microscopy images are displayed in Figure 3B. Clearly, well-defined and radial neurites and astrocytes were formed in the upper part $(\mathrm{H}=50 \sim 150 \mu \mathrm{m})$ but dense and entangled neurites and astrocytes were found in the lower part $(\mathrm{H}=0 \sim 50 \mu \mathrm{m})$. Zoom into the cluster, we also observed astrocyte shuttling inside the cluster (Figure 3C), and a dense contact of axons and astrocytes (Figure 3D), which should have distinct roles in the initialization of synapse formation and regulation of neural activities. 
A NF200/GFAP/DAPI B
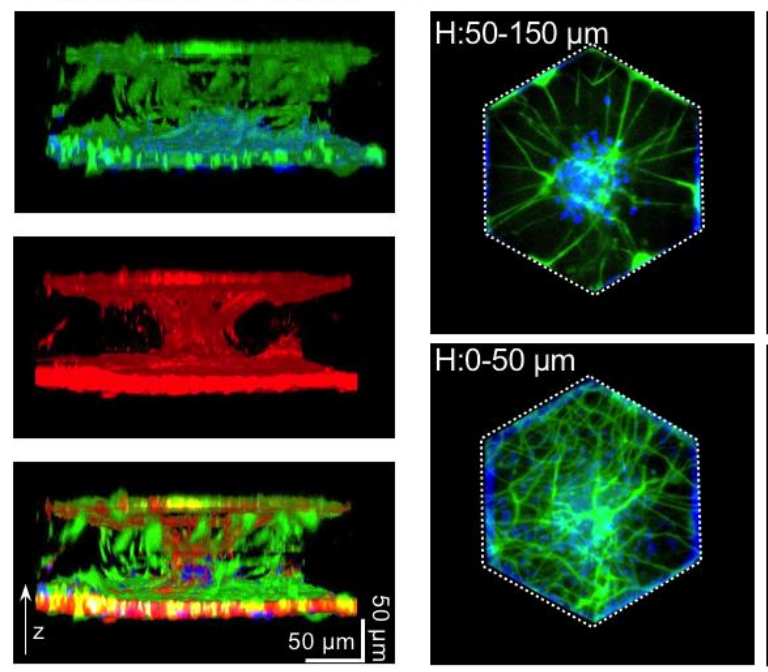

NF200/GFAP/DAPI
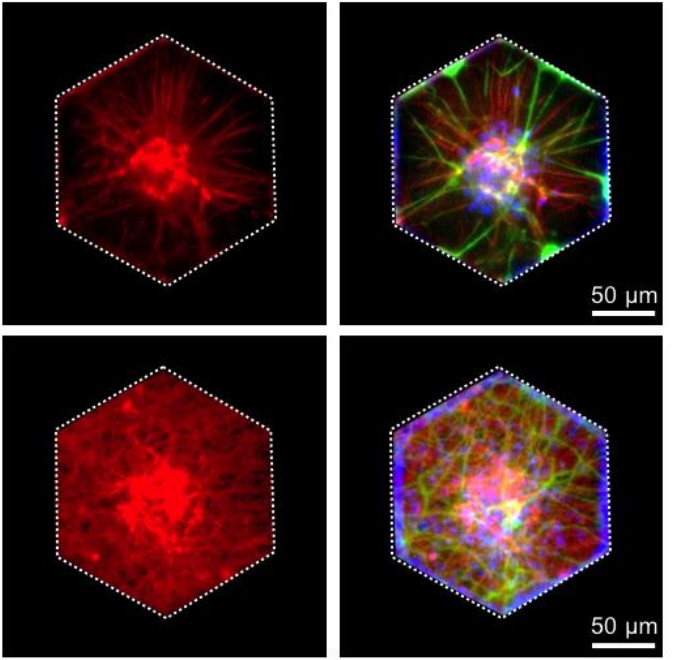

C

D Neural cluster projection
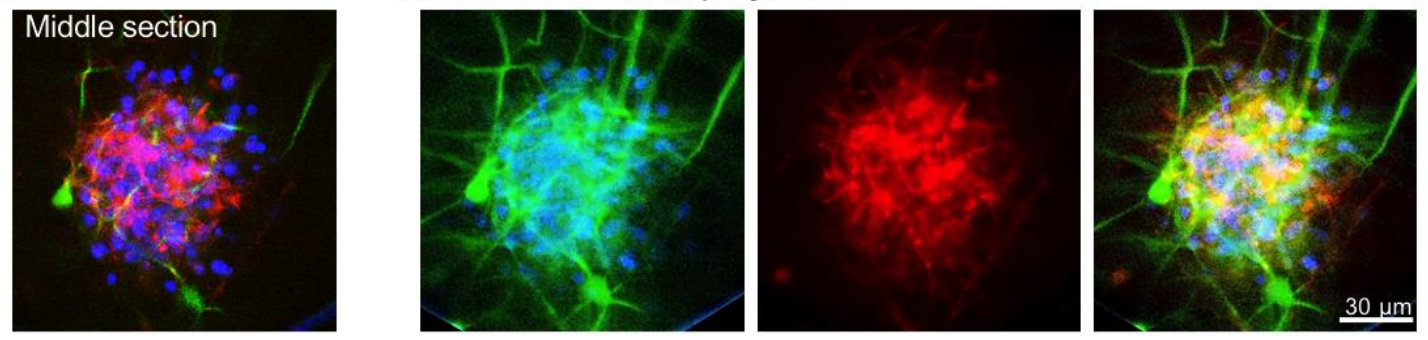

E

NF200/GFAP/DAPI
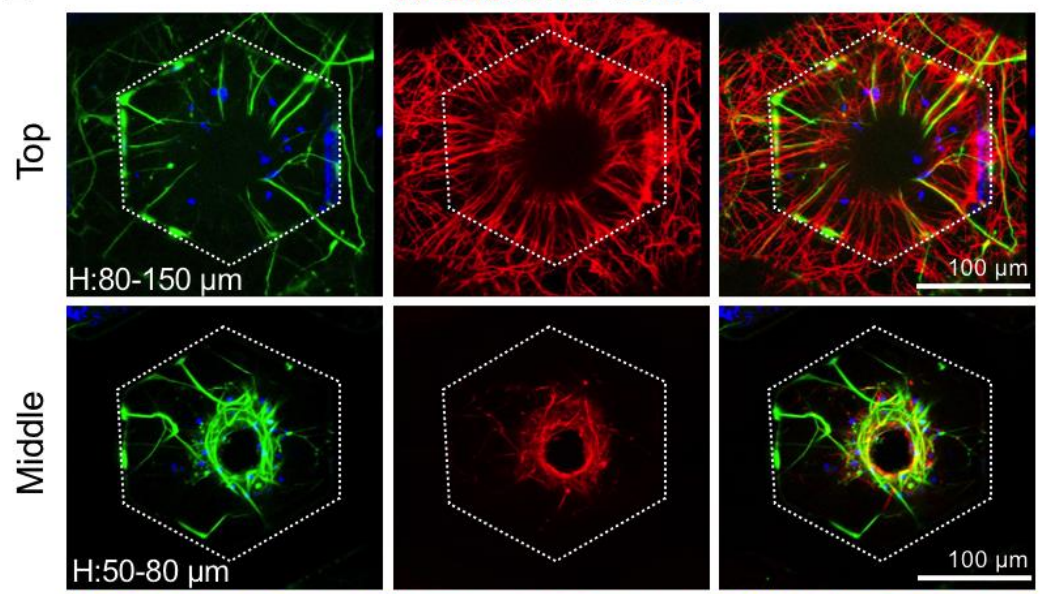

F NF200/GFAP/DAPI
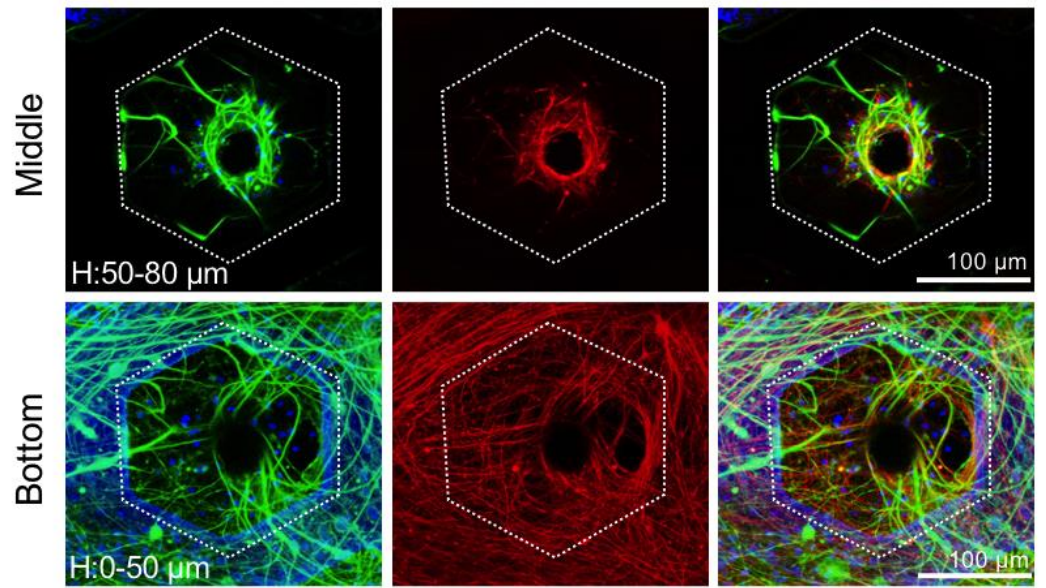
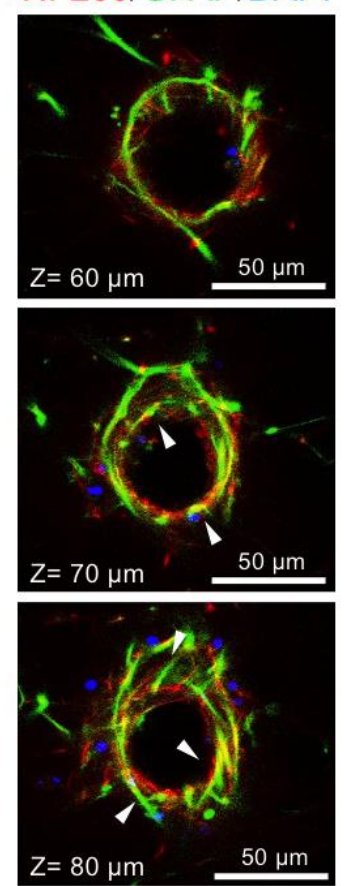
Figure 3. Confocal microscopy images of neurons and astrocytes in honeycomb compartments. Cells were fixed on day 100 and stained by DAPI (blue, nuclei), GFAP (green, astrocytic intermediate filament), and NF 200 (red, neurofilament). A) 3D-reconstruction views of a neural cluster formed inside the honeycomb compartment. B) Regional projection of the corresponding z-stack images. For clarity, the outside of the compartment has been blacked out. C) Zoom in of a cluster, showing astrocyte shuttle inside the cluster, D) Regional projection, showing dense contact of axons and astrocytes. E) Projection of another z-stack image, showing an hourglass-like cluster. F) Formation of astrocytic rings at different height inside the honeycomb compartment.

Statistically, the centralized clusters were observed in $97 \%$ of the honeycomb compartments. In the remaining compartments, surprisingly, we found an hourglass-like organization of neural cells instead of spheroid-like clusters, as shown by the regional projection of confocal microscopy images (Figure 3E, 3F). In the upper region $(\mathrm{H}=80 \sim 150 \mu \mathrm{m}$ ) and out of the center area, astrocytic extensions and neurites were both distributed radially and the neurites were much more than astrocytic extensions. It seems that both astrocytic extension and neurites went to the deeper region. In the middle region $(\mathrm{H}=50 \sim 80 \mu \mathrm{m})$, astrocytic ring structures were found and closely followed by the neurites, suggesting a new type of neural organization which may give rise to an enhanced maturation and an upregulated synaptic formation. In the lower region $(\mathrm{H}=0 \sim 50 \mu \mathrm{m})$, more astrocytic extensions and neurites were found in the frame area but they seem also went to the ring inside the compartment. Such an organization is due probably to the smaller cell number in some of the honeycomb compartments. Although they are statistically few, the same organization can be repeatedly observed, indicating complexity of cell-material interaction as well as variability of threedimensional neural organization. Of note, the astrocytes around spheroid- and hourglass-like clusters were distinctly expanded to the outer space (Figure 3D, 3F), which should be selforganized to maintain the metabolic activities of the clusters. 


\subsection{Maturation of the neural cluster networks}

\subsubsection{Distribution of axons and synapses}

The formation of synapses along axons is a prerequisite for the formation of a neural network. We thus applied immunocytochemistry to observe the distribution of Synapsin I and Tau proteins, which are respectively associated with synaptic vesicles and axons. Figure 4A shows the 3D reconstruction of the confocal microscopy z-stack images of nuclei (blue), Synapsin I (Syn I, green), and Tau (red) inside and around a honeycomb compartment. Clearly,

both Synapsin I and Tau were expressed at a high density all over the hiPSC differentiated neural networks.

The cumulative fluorescence intensities of Synapsin I and Tau on the top and bottom surfaces as well as the interlayer space of the bilayer scaffold are reported in Figure 4B. Here, the frame area surrounding the compartment has been taken into account. As can be seen, the cell number on the top surface is relatively small but both the cumulative fluorescence intensities of Tau and Synapsin I proteins were comparable to that of the bottom surface, suggesting that both nanofiber surfaces were covered with a high density of axons and synapses but the cell bodies were more likely located in the interlayer space. Note that the expressions of Tau and Synapsin I proteins were also significant in the interlayer space, suggesting a matured 3D neural organization. Figure 4C shows regional projections of the confocal microscopy images, indicating that the distributions of the synapses and the axons were highly correlated (Figure 4D) and that both were highly expressed in the center area of the compartment (Figure 4E-F). Moreover, the presence of Synapsin I along the axons which expanded from the cluster to the 
neighboring compartment is a signature of the formation of interconnected cluster network (Figure 4E right).
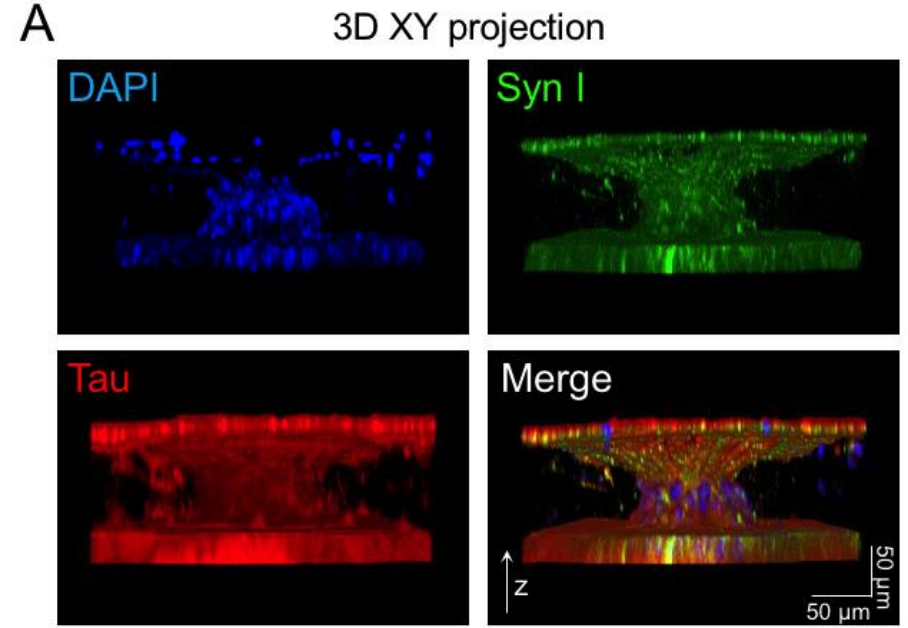

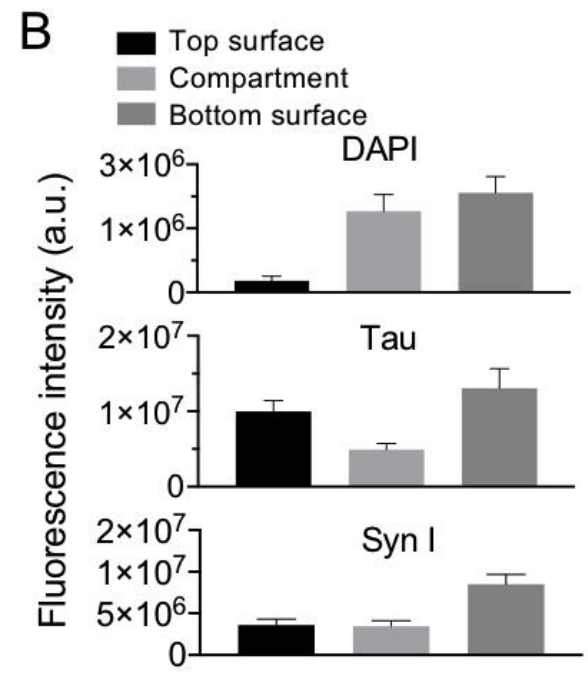

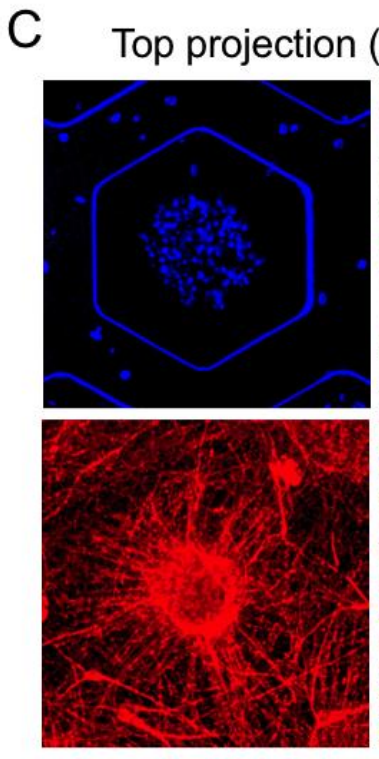

$\mathrm{E}$

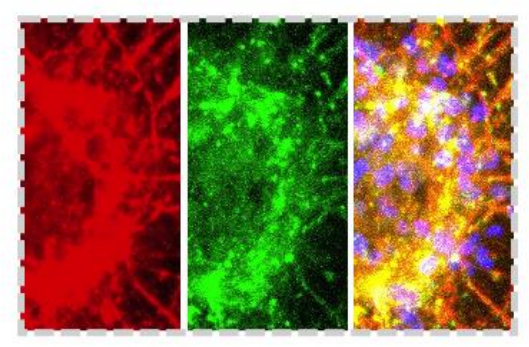

D Bottom projection $(\mathrm{H}: 0-100 \mu \mathrm{m})$
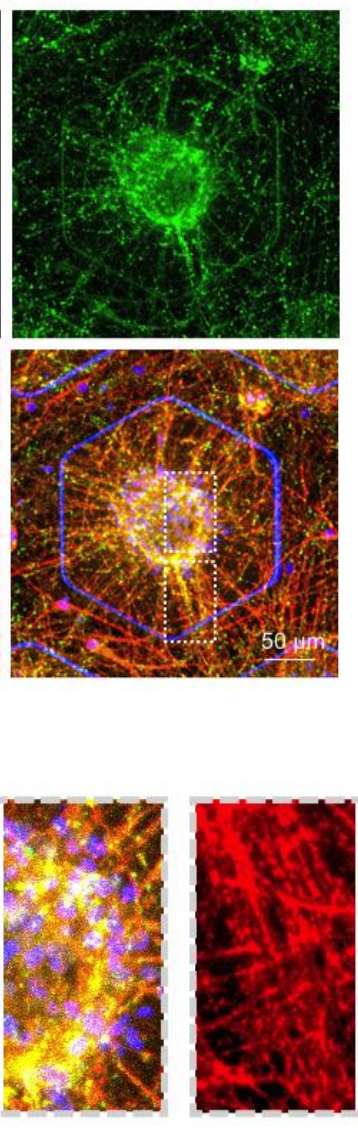
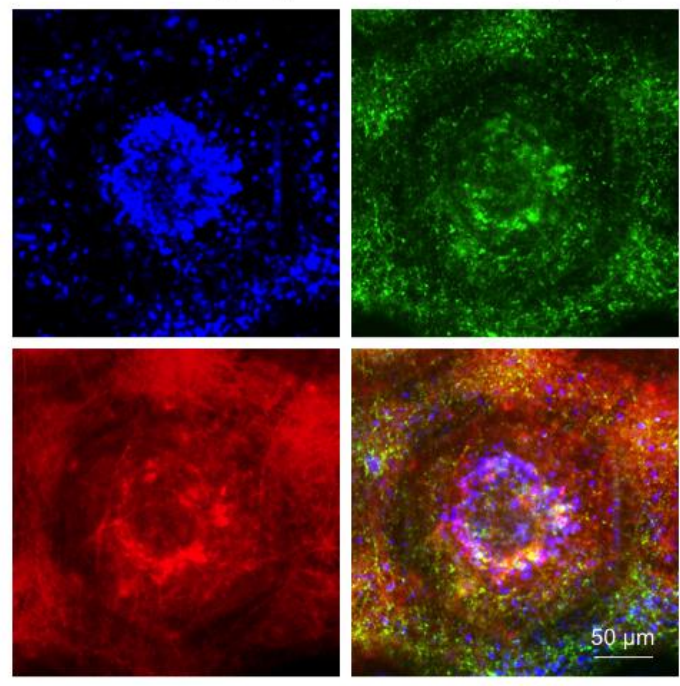

$\mathrm{F}$

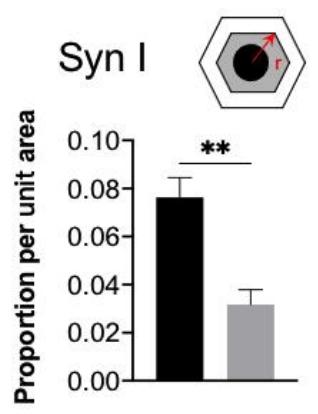


Figure 4. Confocal microscopy images of a neural cluster. Cells were fixed on day 100 and stained by DAPI (blue, nuclei), Synapsin I (Syn I, green), and Tau (red). A) 3D-reconstruction views of a cluster. B) Cumulative fluorescence intensities of DAPI, Synapsin I, and Tau projected in the top surface, compartment, and bottom surface $(n=3$, error bars represent standard error of the mean). C, D) Regional projection of the corresponding Z-stack images, showing a spatial correlation of the synapses and the axons. E) Zoom-in of the images C. F) Regional distribution of synapses deduced from projection of confocal z-stack images in the range between $100 \mu \mathrm{m}$ and $150 \mu \mathrm{m}$, indicating the high-level expression in the center area of the compartment $(\mathrm{n}=3, \mathrm{P}<0.01$ student-t-test). Black area, $\mathrm{r} \leqslant 50 \mu \mathrm{m}$.

\subsubsection{Intralayer correlation of the neural activities}

To demonstrate the intralayer correlation of the neural activities of the 3D neural networks, we focused on the $\mathrm{Ca}^{2+}$ waves recorded on the top of the scaffold (Figure 5A). The ROIs are also marked where ROI 1 covers the whole region of a neural cluster, ROI 2-5 covers the somas of individual bridging cells, and ROI 6 corresponds to the neighboring inter-compartment region. The time traces of $\Delta \mathrm{F} / \mathrm{F}$ are presented in Figure $5 \mathrm{~B}$, where the correlation between different ROIs is clearly observed as highlighted with red and blue bands, demonstrating that the electrical coupling of the neural network over the whole observed area. Figure 5C shows the series of epifluorescence images within one of those highlighted events in Figure 5B, with false colors coding the fluorescence intensity. The fluorescence intensity increased during $1 \mathrm{~s}$ (Figure 5D) on the neurites connected to the neural cluster (white arrowheads) and on the neurites surrounding the neural cluster across the radially projected neurites (black arrowheads), as well as on the neural cluster, showing the electrical signal propagation in all directions over the microframe.

At the bottom of the scaffold, the cell growth and the neural network formation were guided by the gelatin nanofibers. Among them, 22 regions marked by yellow dots were selected (Figure 5E) for detailed analysis by using Fluorescence Single Neuron and Network Analysis Package (FluoroSNNAP) ${ }^{45}$. The connectivity between neuron pairs is indicated with the red lines. The 
fluorescence intensity peak events of the cells were then plotted as a function of time (Figure 5F) to illustrate the coactivity of the network. Here, the green dots are data from the neurons of the same compartment, while the pink dots are from the neighboring compartment. Then, a synchronization matrix for pair-wise phase-synchronization indices could be depicted as a heatmap (Figure 5G), revealing that the electrical activities of these cells are highly synchronized with a global synchronization index of 0.83 . 
A

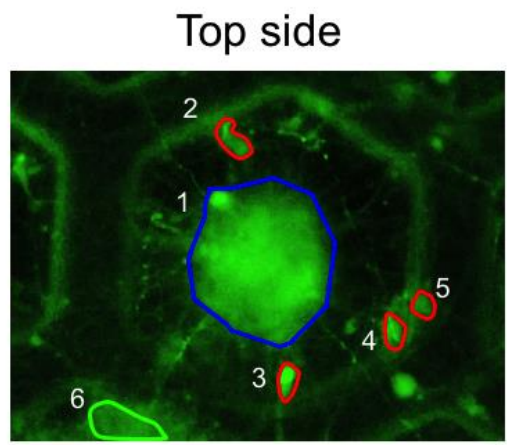

B

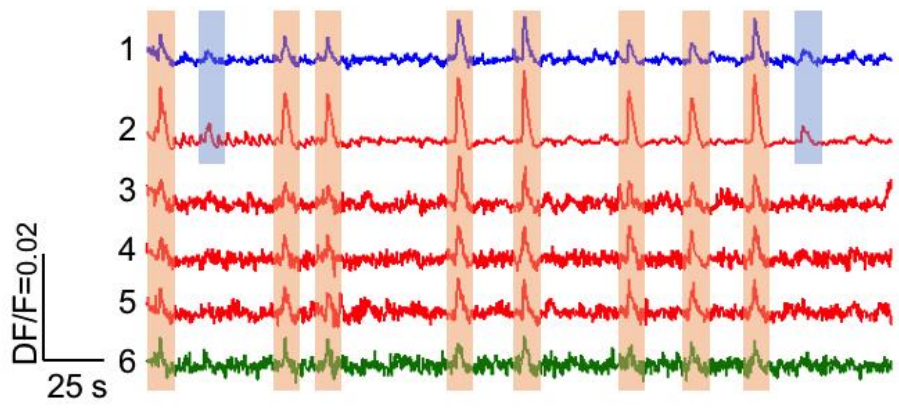

C

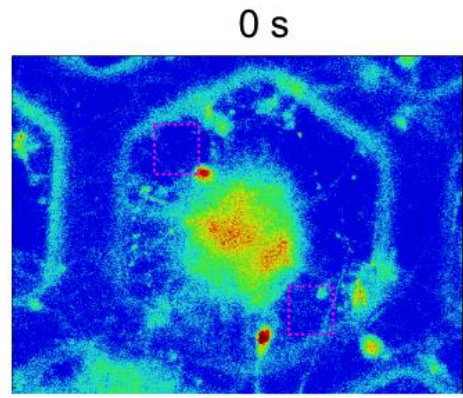

$0.5 \mathrm{~s}$
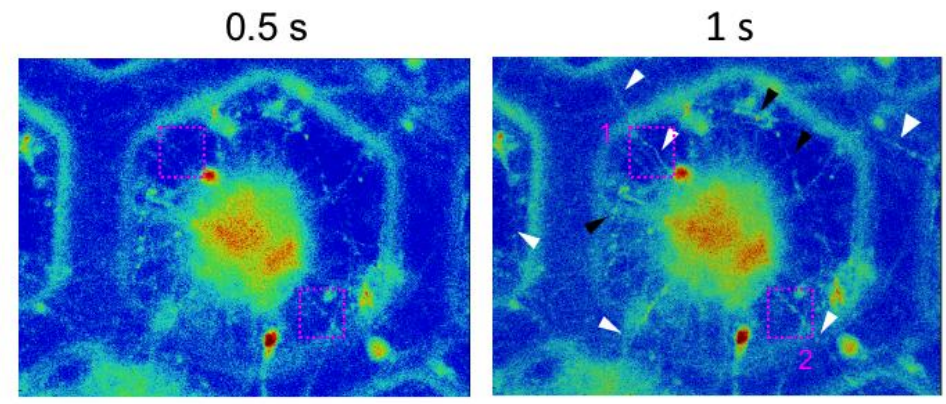

90

D

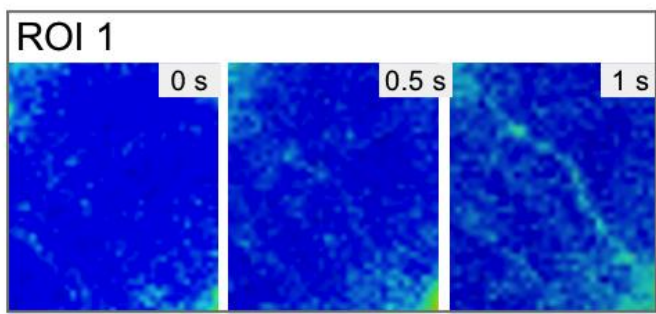

E

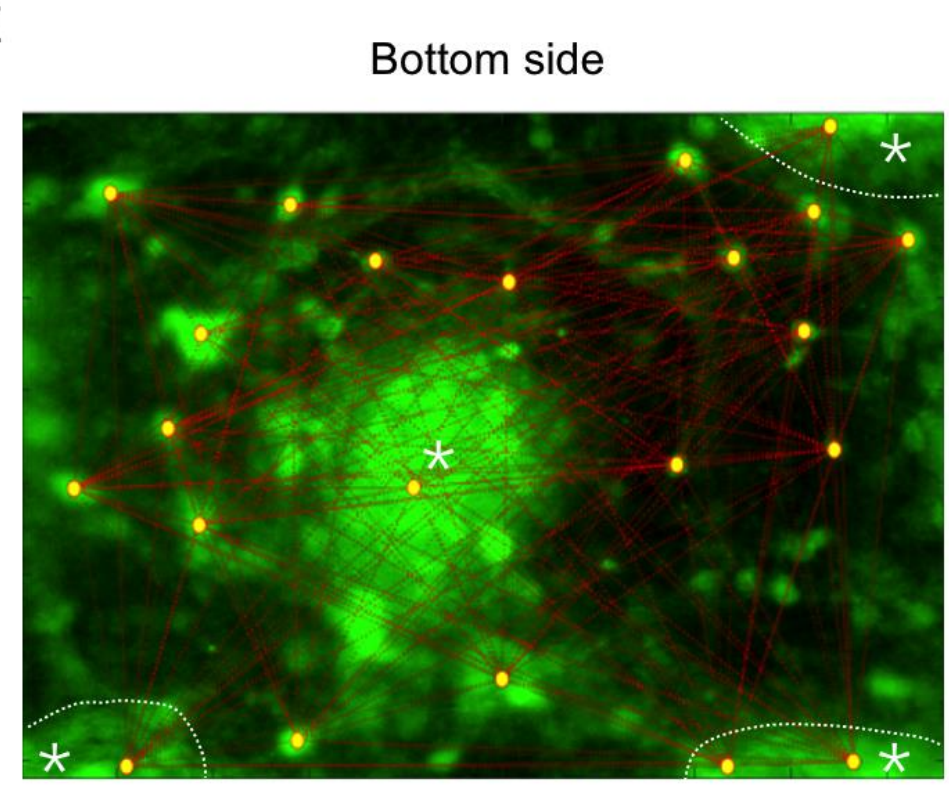

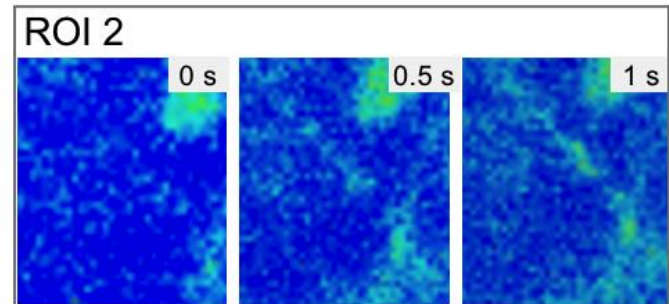

F

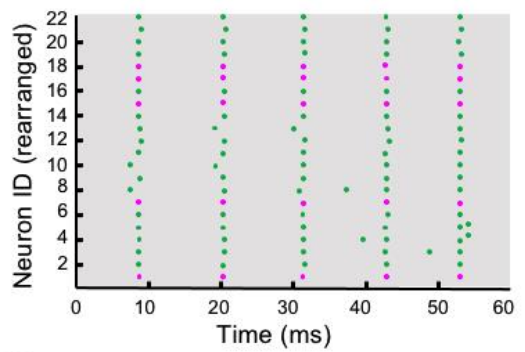

G Global synchronization index: 0.833675

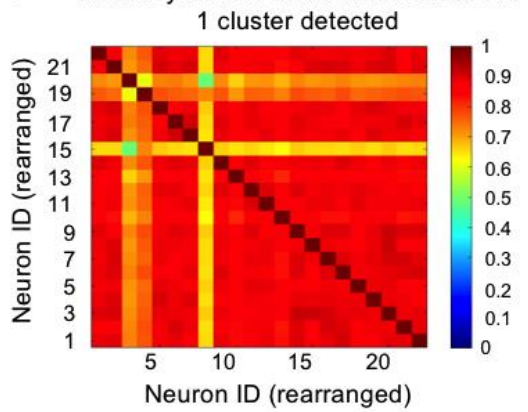


Figure 5. Calcium images showing intralayer correlation of the neural activities. A) Epifluorescence microscopy image of the top side after loading $\mathrm{Ca}^{2+}$ indicator, Fluo-4 AM. ROIs for fluorescence intensity measurements are indicated. ROI 1 covers the whole area of the neural cluster. ROI 6 covers the area of the neighboring neural cluster $\mathrm{B}$ ) Time traces of $\Delta \mathrm{F} / \mathrm{F}$ in ROIs shown in A. Synchronized peaks are highlighted with red bands (total synchronization) and blue bands (partial synchronization). Correlated spikes accorded at ROI 1-6. C) The fluorescence intensity increased during $1 \mathrm{~s}$ on the neurites connected to the neural cluster and the neurites surrounding the neural cluster, indicated by white and black arrowheads, respectively, as well as on the neural cluster. D) Zoom-in of image $\mathrm{C}$ showing correlated neural activities in two sides of a honeycomb compartment. E) Epifluorescence microscopy image of the neural cluster with a focal plane at the bottom. Twenty-two cell bodies identified by FluoroSNNAP program are indicated with yellow dots. Functional connectivity detected by the program between neuron pairs is indicated with red lines. F) Raster plot of fluorescence intensity peaks in 22 ROIs shown in $\mathrm{E}$, arranged depending on their contribution to the network activity (green dots: this compartment, pink dots: the surrounding compartments). G) Synchronization matrix with a color map for pair-wise phase synchronization indices, generated by FluoroSNNAP program.

\subsubsection{Interlayer correlation of the neural activities}

$\mathrm{Ca}^{2+}$ transient images were recorded to trace $\mathrm{Ca}^{2+}$ ion flux associated with electrical activity of neurons using Fluo-4 acetoxymethyl ester (Fluo-4 AM) as an indicator. As shown above, the cell density at the bottom of the scaffold is higher than that of the top. The sample was thus flipped upside down for more clear observation of the calcium waves at different focal planes with an epifluorescence microscope. Figure 6A shows typical epifluorescence microscopy images of the neural network at different focal planes, where the ROIs are marked with numbers and orange dots. These ROIs are also reported in Figure 6B for a projection view. Note that ROI 6 corresponds to the area covering the whole area of the neural cluster, whereas ROI 7-12 represent distinct cell bodies inside ROI 6. The sample was scanned along the z-axis to cover the whole scaffold and the ROIs were divided into 5 height levels, (h1, h2, h3, h4, h5 = 0, 21, 54, 93, $102 \mu \mathrm{m}$ ), at which the cells were best focused (Figure 6C). Here, h1 and h5 correspond to the top and bottom of the bilayer scaffold and the image in Figure $6 \mathrm{~B}$ was taken at h3. On the same focal 
plane, $\mathrm{Ca}^{2+}$ waves were recorded every $500 \mathrm{~ms}$ during $5 \mathrm{~min}$. Normalized fluorescence intensity relative to the initial value, $\Delta \mathrm{F} / \mathrm{F}$, in each $\mathrm{ROI}$ was traced and presented according to the height of the cell position in Figure 6D. Interestingly, the recorded signals from different ROIs were correlated throughout the whole height of the scaffold as highlighted with the red bands or partially as highlighted with the gray and blue bands. These results revealed an interlayer correlation of the neural activities of the 3D neural networks formed in our bilayer nanofiber scaffold.
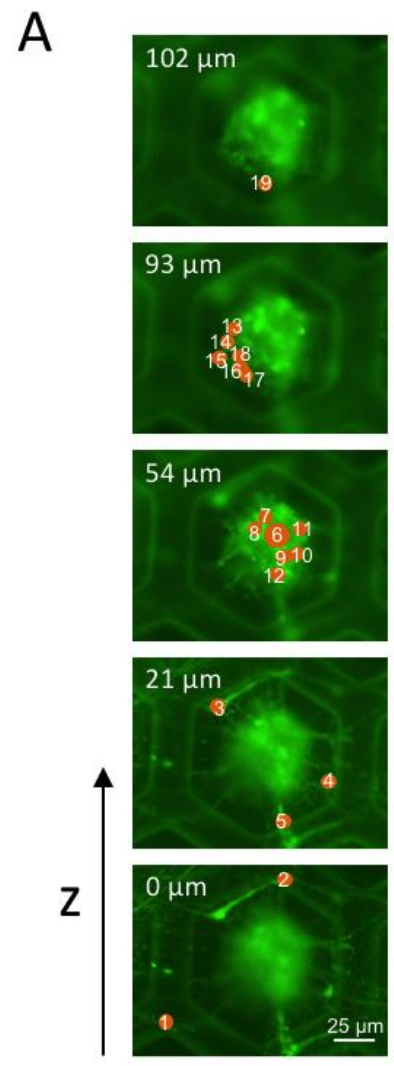

\section{B}
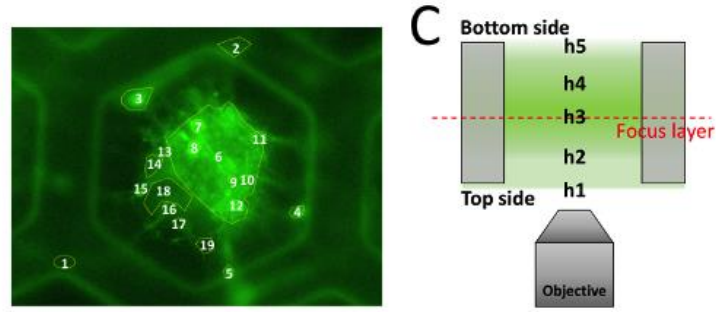

h5: $102 \mu \mathrm{m}$

h4: $93 \mu \mathrm{m}$

h3: $54 \mu \mathrm{m}$

h2: $21 \mu \mathrm{m}$

h1: $0 \mu \mathrm{m}$

D

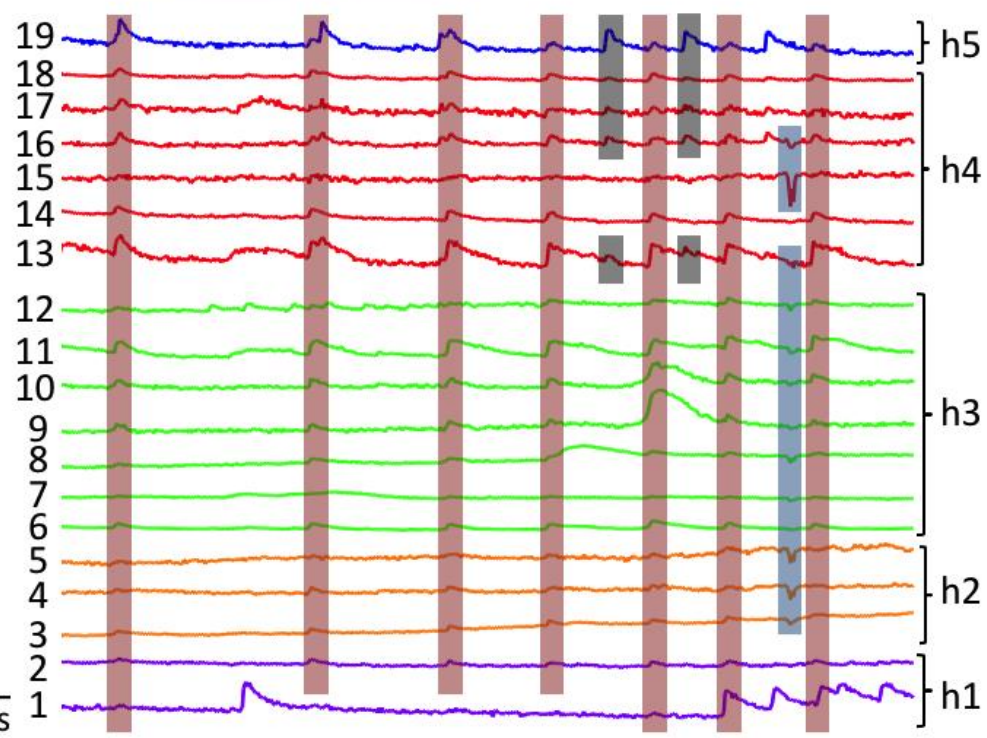

Figure 6. Calcium images showing interlayer correlation of the neural activities. A)

Epifluorescence microscopy images of the neural cluster at different focal planes. The number of ROIs shown in A are marked with orange dots when best focused. B) A summary of the ROIs in an epifluorescence microscopy image of the neural cluster observed on the focal plane at $54 \mu \mathrm{m}$. C) schematic of the 5 focal planes. D) Time traces of normalized fluorescence intensity relative 
to the initial value, $\Delta \mathrm{F} / \mathrm{F}$, in ROI 1-19 presented according to the height of the cell position. Synchronized peaks are highlighted with red bands (total synchronization, including the neural cluster) and gray or blue bands (partial synchronization).

\subsubsection{Effects of the seeding cell density}

For comparison, similar experiments have been performed with small seeding cell densities. Although the cell aggregation still occurred in each honeycomb compartment, the other effects including the neural cluster formation and the correlated neural activities could not be clearly observed. Figure S5 shows the results obtained with a seeding density of $2.0 \times 10^{5}$ NPCs per side of the bilayer scaffold, which is 1.5 times less than the previous one. Clearly, the differentiating NPCs can still lead to the formation of 3D neural networks (Figure S5A and Figure S5B, left) and the differentiated neurons and astrocytes are still coupled (Figure S5B, right). Although a small group of cells on the frame showed correlated neural activities (ROI 1-5), the signals were weak and the small cluster inside the compartment had no correlation of the neural activities with surrounding cells (Figure S5C), due to the insufficient number of neurons and connections.

\subsection{Co-culture of the neural networks with endothelial cells}

After incorporating the ABM into the bottom layer nanofibers of the scaffold (Figure 7A), NPCs were firstly loaded on the top layer of the scaffold and then differentiated for 8 weeks toward neural networks (Figure 7B). During this period, the majority of the cells remained on the top of the scaffold without being attracted downwards except a limited number of the cells inside the honeycomb compartment. Afterward, brain microvascular endothelial cells (hCMEC/D3 
cells) were added on the backside of the scaffold for the formation of endothelium on ABM. The endothelial cells got confluent quickly at day 0 (Figure $\mathrm{C}$, right, day 0). After incubation for 7 days, the endothelial cells remained but dark shadows appeared in the center area of the compartment, indicating that the presence of the endothelium influences the spatial organization of the neural network (Figure 7C, right). No such effect was observed with the sample without hCMEC/D3 cells (Figure 7C, left). The neural cells were then stained with DAPI (blue), GFAP (green), and NF 200 (cyan) for nuclei, astrocytes, and neurons, respectively. In addition, the actin cytoskeleton was also stained by fluorescent phalloidin (red). As shown in Figure 7D, hCMEC/D3 cells stained in red formed dense cell layer, neurons stained in cyan mostly remained on the top of the scaffold, and some astrocytes elongated vertically with remarkable end-feet in the contact area with the endothelium (Figure 7D-F). The neural cells on the top and the endothelial cells on the bottom of the scaffold are also displayed in Figure 7G and 7H, showing the network formation of astrocytes (GFAP, green) and neurons (NF200, cyan) and the formation of a confluent hCMEC/D3 cells layer. Altogether, our results provided a proof of concept of in vitro NVU modeling. 
A
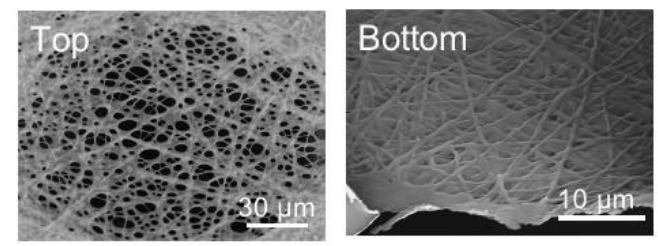

C

- hCMEC/D3 cells

Day 1
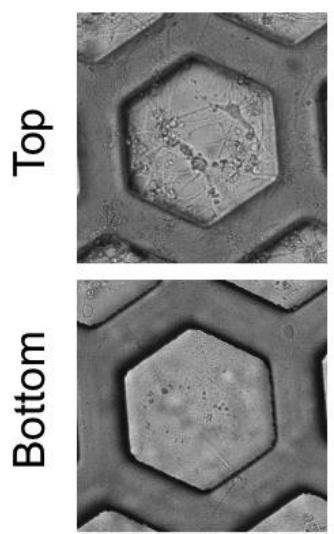

D

3D reconstruction

GFAP/F-actin/NF200/DAPI

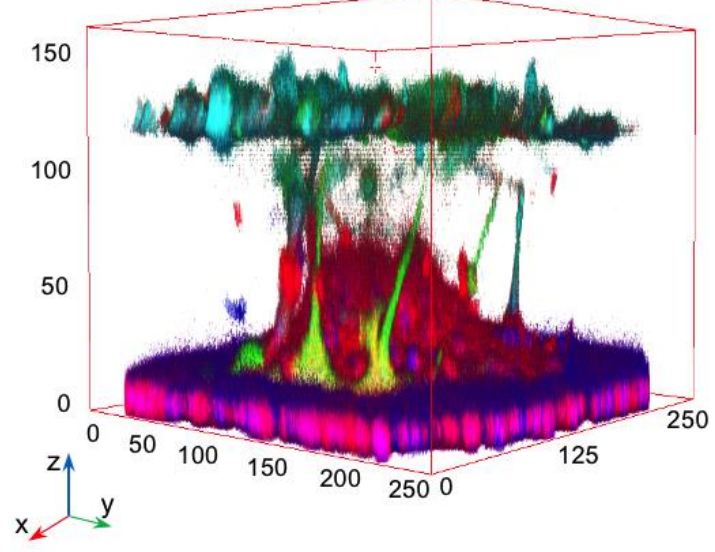

B
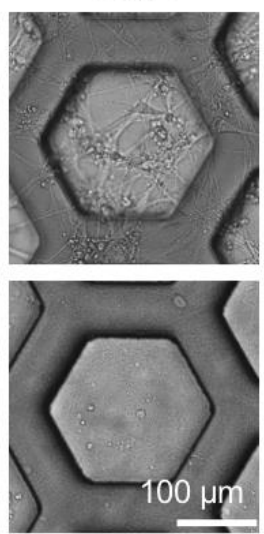

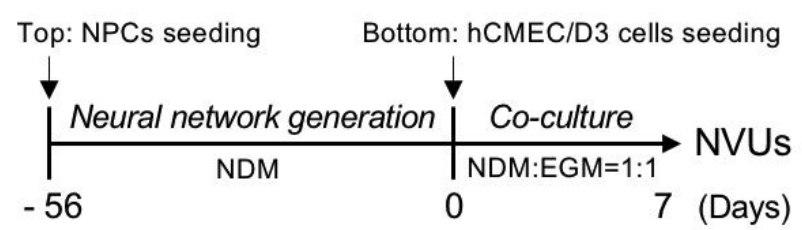

$+\mathrm{hCMEC/D3}$ cells

Day 0

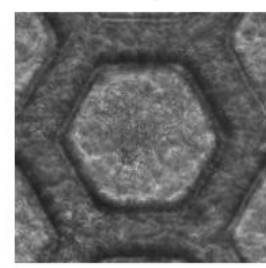

Day 1

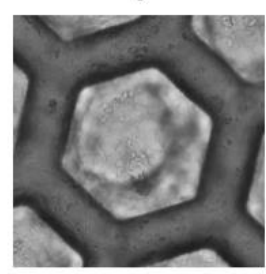

Day 7
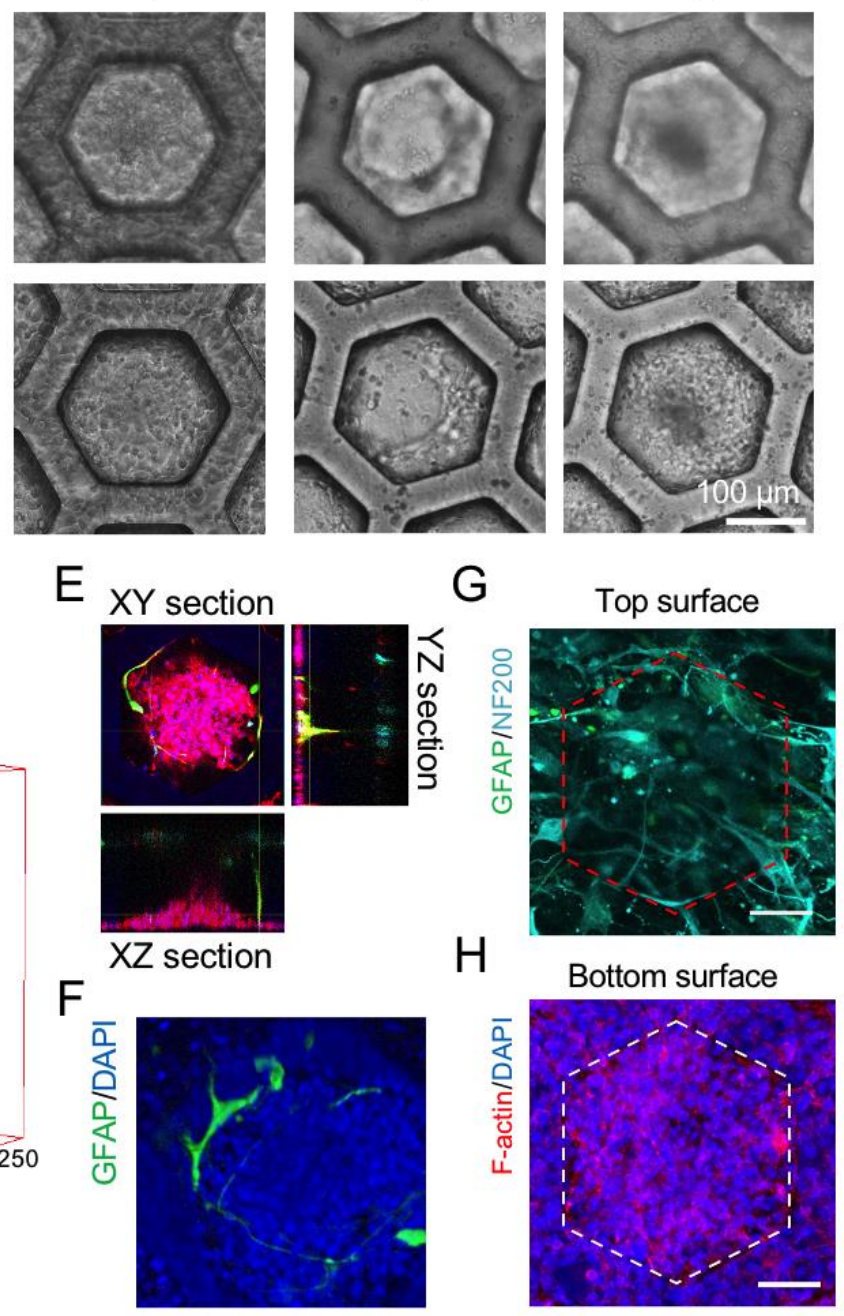

Figure 7. Coculture of neural cells and brain microvascular endothelial cells with an ABM incorporated bilayer nanofiber scaffold. A) SEM images of the top layer (left) and the bottom layer (right) of the scaffold. B) Cell processing steps. C) Top and bottom layer focused phasecontrast images of the cells without and with coculture with hCMEC/D3 cells after differentiation for 56 days with the scaffold. Cells were fixed after coculture for 7 days and stained by DAPI (blue, nuclei), F-actin (red, cytoskeleton), GFAP (green, astrocytes), and NF 200 (cyan, neurons). D) 3D-reconstruction view of the immunostaining images of an NVU. E) 
Orthogonal view of a reconstructed image using the same z-stack images. F) 3D views of nucleus and astrocytes labeled images. G) Top view of the neural network (cyan, neurons; green, astrocytes). Scale bars, $50 \mu \mathrm{m}$. H) Bottom view of the cells on an ABM. Scale bars, $50 \mu \mathrm{m}$.

\section{DISCUSSION}

Engineered microsystems can be used for spheroids and organoids studies ${ }^{50}$ as well as organ-on-a-chip investigations ${ }^{51}$. Here, we developed a sophisticated but yet reliable system for 3D guided self-organization of $\mathrm{CNN}$ and, after incorporating an artificial basement membrane, coculture of $\mathrm{CNN}$ and endothelial cells. This has been based on a hierarchic design and multitechnological processing, involving lithography and pattern transfer, electrospinning, chemical crosslinking, and self-assembling. The advantage of the lithography techniques relies on its flexibility in mask and mold design so that the geometry parameters of honeycomb microframe (size, wall width and thickness) can be changed, meaning that it is possible to design CNNs with different cluster size and different inter-cluster distance. Electrospinning is a simple and widely technique to produce nanofibers. It can work with different types of materials or materials composites and result generally in a fibrous morphology of culture substrate often used for adherent cell culture, tissue engineering, and more recently for stem cell culture and differentiation ${ }^{42,43,52-55}$. The advantage of this technique also relies on its flexibility to control the diameter and porosity of the nanofibers. It is believed that the morphology of nanofibers is benefitable to the formation of neural tissues ${ }^{56,57}$. More recently, we demonstrated that both cardiomyocytes and motor neurons could be differentiated from hiPSCs on compartmented monolayer of crosslinked nanofibers ${ }^{42,43}$. Primary neural cells could also be cultured on such a device, showing in-vitro like astrocytic morphology and enhanced neural activities compared to that on a glass slide ${ }^{52}$. Finally, self-assembling of biomolecules is widely used for surface 
functionalization of culture substrates and tissue scaffolds, as the last step of multitechnological fabrication of hierarchic structures ${ }^{58}$. In our case, it allows spontaneous formation of ultrathin layers in the nanofiber pores by dehydrating a protein solution mixture (collagen IV and laminin). This could be possible due to large surface tension of the layer and self-assembling of superimposed molecular networks of collagen IV and laminin.

A unique advantage of the bilayer nanofiber scaffold is the low effective Young's modulus of the monolayer of gelatin nanofibers. Let us consider a network formed with hexagonal cells of wall width and thickness $d$ and segment length $l$, and Young's modulus of $E_{s}$. Within the zeroorder approximation, the effective in-plane Young module is equal to $\frac{4}{\sqrt{3}} E_{S}\left(\frac{t}{l}\right)^{3}$, while the effective out-of-plane Young module is equal to $\frac{2}{\sqrt{3}} E_{S}\left(\frac{t}{l}\right) \cdot{ }^{59}$ If $t=0.5 \mu \mathrm{m}, l=5 \mu \mathrm{m}$, and $E_{s}=1.35$ $\mathrm{MPa}$, we obtained an effective in-plane Young's modulus $\sim 13.14 \mathrm{kPa}$, and an effective out-ofplane Young's modulus $\sim 156 \mathrm{kPa}$. Here, the gelatin nanofibers were submerged in a culture medium so that a Young's modulus of wetted gelatin was used. Experimentally, this has been verified by two types of assays. The first one was similar to that described in ${ }^{46}$. The monolayer of nanofibers was integrated into a microfluidic device with a thin PDMS cover layer, the pressure induced deflection of the membrane in a honeycomb frame was determined. Similarly, the deflection of the same PDMS layer was determined. After some algebra, an effective Young's modulus was deduced. The second method relied on AFM measurement which gave an effective Young's modulus in the same order as above discussed. On the other hand, the monolayer of crosslinked nanofibers was bonded on a microframe so that the stress in the honeycomb center should be smaller than that near the edge since the total stress is the sum of the average tensile stress and the bending stress which is maximal at the edge ${ }^{60}$. In other words, 
the stiffness of the nanofiber membrane is inhomogeneous and it is the smallest in the center of the honeycomb frame. Such a stiffness gradient promotes cell migration to the center of the membrane whereas the accumulation of the cells and in particular the infiltration of cells into the interlayer space leads to not only a strong cell-nanofibers interaction but also a strong effect of self-organization ${ }^{61,62}$, as observed in Figure 2. More systematic studies will be carried to elucidate the influence of the nanofiber stiffness on the cell culture behaviors.

We would like to mention that the goal of this work is to provide proof of concept of guided self-organization of cortical neural networks by using micro-engineered scaffolds. This goal has been achieved through the use of hiPSCs and bilayer nanofiber scaffold, offering a reliable way to investigate systematically the neural network functions by varying, for example, the size of the clusters and the distance between two clusters. In Figure 3C, 4A, and 4C, we can see that the cell aggregation in a cluster is not tightly packed and that the neural cells inside a cluster have a center-to-center distance of $\sim 10 \mu \mathrm{m}$. Roughly speaking, with a cylindric cluster of $75 \mu \mathrm{m}$ diameter and $50 \mu \mathrm{m}$ height, a cell number of $\sim 250$ can be estimated. The question arises whether the cluster size and the inter-clusters distance can be optimized. This can be studied by modifying the geometry of the honeycomb microframe and the cell seeding density. In this regard, it would be interesting to correlate the scaffold design with theories in order to understand more clearly how a neural cluster work and how they communicate each other in a large context of signal processing. Finally, as the majority of the cells were located on the bottom layer of the scaffold due to gravitation, it would be interesting to reduce this effect to achieve a more symmetric cell distribution.

We would also like to mention that in the case of bilayer scaffold with ABM, neural cells also infiltrated in the interlayer space of the scaffold, but the resulted neural clusters were 
different from that with a scaffold without ABM, due probably to insufficient infiltration knowing that here NPCs cells were loaded only from upside of the scaffold. Due probably to the presence of a limited number of neural cells in this space, the deformation of the nanofibers was not observed neither. This could be improved by increasing the pore size of the nanofibers and increasing the cell seeding density, for example. Nevertheless, the formation of large astrocytic endfeet observed in the work is significant since it is a clear signature of spontaneous formation of the brain-blood barrier which is required for sustaining neural activities. Factors released from endothelial cells might induce the extension of astrocytic endfeet. We have also to mention that a cell line of microvascular endothelial cells was used which might be replaced with hiPSC derived cells using the source of cell source. Similarly, microglial cells derived from the same hiPSCs could be added. Different types of assays can be envisaged but mostly importantly both generated CNN- and NVU-like systems have to be subjected to more detailed characterization by using such as multielectrode array (MEA) system for electrophysiological stimulation and data analysis. Furthermore, the scaffold with functional CNN or NVU like structures can be integrated into microfluidic devices for systematic investigations under both physiological and pathological conditions, with and without chemical and physical stimuli ${ }^{63,64}$. Last but not least, the differentiation processes have to be automatized in order to achieve a sustainable progress, although this has been done for proof-of-concept demonstration ${ }^{65}$.

\section{CONCLUSION}

We developed a method for in vitro modeling of cortical neural networks with a scaffold made of honeycomb microframe covered with a monolayer of crosslinked gelatin nanofibers, 
with and without incorporating a reconstituted basement membrane. Inter-connected 3D neural clusters were generated due to guided self-organization of differentiating NPCs. We observed high-level expressions of the neuron and astrocyte-specific proteins as well as high-density synaptic vesicles along axons. The calcium transient images showed spatially correlated neural activities, revealing the importance of 3D organization of the network. Finally, we cocultured the neural network with endothelial cells and observed vertical astrocytes with large endfeet in the contact area backside the endothelium. Altogether, this proof of concept provides a view on the potential for use of nanofiber-scaffolds in studies of cortical neural networks.

\section{ASSOCIATED CONTENT}

\section{Supporting Information}

The Supporting Information is available free of charge on the ACS Publication website. The Supporting Information includes the schematic of fabrication process of the scaffold (Figure S1A), statistics of the pore area of the nanofiber layer (Figure S1B), the images of the development of neural clusters (Figure S2), nomination of the region of interest (Figure S3), analysis of the population of neurons and astrocytes on the scaffold (Figure S4) and results of the small neural networks in the scaffold (Figure S5).

\section{AUTHOR INFORMATION}

\section{Corresponding Author}

*Yong CHEN: yong.chen@ens.psl.eu; * Ayako YAMADA: ayako.yamada@ens.psl.eu 


\section{Author Contributions}

The manuscript was written through contributions of all authors. All authors have given approval to the final version of the manuscript.

\section{Notes}

The authors declare no competing financial interest.

\section{ACKNOWLEDGMENT}

B. H. has been supported by a Guangzhou Elite Fellowship. The authors thank Prof.

Vincent Torres from Scuola Internazionale Superiore di Studi Avanzati (Iatly), Dr. Marie-Claude Potier from Institute of Brain and Sorbonne University (France), Jean-Michel Peyrin and Teng Pan at the Institut de Biologie Paris-Seine for fruitful discussions. This work has been supported by grants from Agence Nationale pour la Recherche (ANR-17-CE09-0017, ANR-19-CE180009-01), Région Ile-de-France (DIM-ELICIT), PSL-valorization (program pre-maturation), Carnot IPGG, and European Commission Cost Action project BIONECA (CA 16122). This work has also received the support of "Institut Pierre-Gilles de Gennes" (laboratoire d'excellence, "Investissements d'avenir" program ANR-10-IDEX-0001-02 PSL and ANR-10LABX-31.).

\section{REFERENCES}

(1) Lui, Jan H.; Hansen, David V.; Kriegstein, Arnold R. Development and Evolution of the Human Neocortex. Cell. 2011, 146, 18-36.

(2) Shapson-Coe, A.; Januszewski, M.; Berger, D. R.; Pope, A.; Wu, Y.; Blakely, T.; Schalek, R. L.; Li, P. H.; Wang, S.; Maitin-Shepard, J.; Karlupia, N.; Dorkenwald, S.; Sjostedt, E.; Leavitt, L.; 
Lee, D.; Bailey, L.; Fitzmaurice, A.; Kar, R.; Field, B.; Wu, H.; Wagner-Carena, J.; Aley, D.; Lau, J.; Lin, Z.; Wei, D.; Pfister, H.; Peleg, A.; Jain, V.; Lichtman, J. W. A connectomic study of a petascale fragment of human cerebral cortex. bioRxiv. 2021, 446289.

(3) Camandola, S.; Mattson, M. P. Brain metabolism in health, aging, and neurodegeneration. The EMBO Journal. 2017, 36, 1474-1492.

(4) McConnell, H. L.; Kersch, C. N.; Woltjer, R. L.; Neuwelt, E. A. The Translational Significance of the Neurovascular Unit *. J. Biol. Chem. 2017, 292, 762-770.

(5) Banks, W. A. From blood-brain barrier to blood-brain interface: new opportunities for CNS drug delivery. Nat. Rev. Drug Discovery. 2016, 15, 275-292.

(6) Yu, X.; Ji, C.; Shao, A. Neurovascular Unit Dysfunction and Neurodegenerative Disorders Front. Neurosci. 2020, 14, 334.

(7) Yamazaki, Y.; Kanekiyo, T. Blood-Brain Barrier Dysfunction and the Pathogenesis of Alzheimer's Disease. Int. J. Mol. Sci. 2017, 18, 1965.

(8) Frega, M.; Tedesco, M.; Massobrio, P.; Pesce, M.; Martinoia, S. Network dynamics of 3D engineered neuronal cultures: a new experimental model for in-vitro electrophysiology. Sci Rep. 2014, 4, 5489.

(9) Ulloa Severino, F. P.; Ban, J.; Song, Q.; Tang, M.; Bianconi, G.; Cheng, G.; Torre, V. The role of dimensionality in neuronal network dynamics. Sci Rep. 2016, 6, 29640.

(10) Lee, C. S.; Leong, K. W. Advances in microphysiological blood-brain barrier (BBB) models towards drug delivery. Curr. Opin. Biotechnol. 2020, 66, 78-87. 
(11) Bhalerao, A.; Sivandzade, F.; Archie, S. R.; Chowdhury, E. A.; Noorani, B.; Cucullo, L. In vitro modeling of the neurovascular unit: advances in the field. Fluids Barriers CNS. 2020, 17, 22.

(12) Nikolakopoulou, P.; Rauti, R.; Voulgaris, D.; Shlomy, I.; Maoz, B. M.; Herland, A. Recent progress in translational engineered in vitro models of the central nervous system. Brain. 2020, $143,3181-3213$.

(13) Koroleva, A.; Deiwick, A.; El-Tamer, A.; Koch, L.; Shi, Y.; Estévez-Priego, E.; Ludl, A. A.; Soriano, J.; Guseva, D.; Ponimaskin, E.; Chichkov, B. In Vitro Development of Human iPSCDerived Functional Neuronal Networks on Laser-Fabricated 3D Scaffolds. ACS Appl. Mater. Interfaces. 2021, 13, 7839-7853.

(14) Ahn, S. I.; Sei, Y. J.; Park, H.-J.; Kim, J.; Ryu, Y.; Choi, J. J.; Sung, H.-J.; MacDonald, T. J.; Levey, A. I.; Kim, Y., Microengineered human blood-brain barrier platform for understanding nanoparticle transport mechanisms. Nat Commun. 2020, 11, 175.

(15) Lee, S. W. L.; Campisi, M.; Osaki, T.; Possenti, L.; Mattu, C.; Adriani, G.; Kamm, R. D.; Chiono, V. Modeling Nanocarrier Transport across a 3D In Vitro Human Blood-Brain-Barrier Microvasculature. Adv. Healthc. Mater. 2020, 9, 1901486.

(16) Cho, H.; Seo, J. H.; Wong, K. H. K.; Terasaki, Y.; Park, J.; Bong, K.; Arai, K.; Lo, E. H.; Irimia, D. Three-Dimensional Blood-Brain Barrier Model for in vitro Studies of Neurovascular Pathology. Sci Rep. 2015, 5, 15222.

(17) Maoz, B. M.; Herland, A.; FitzGerald, E. A.; Grevesse, T.; Vidoudez, C.; Pacheco, A. R.; Sheehy, S. P.; Park, T.-E.; Dauth, S.; Mannix, R.; Budnik, N.; Shores, K.; Cho, A.; Nawroth, J. C.; Segrè, D.; Budnik, B.; Ingber, D. E.; Parker, K. K. A linked organ-on-chip model of the human 
neurovascular unit reveals the metabolic coupling of endothelial and neuronal cells. Nat. Biotechnol. 2018, 36, 865-874.

(18) Canfield, S. G.; Stebbins, M. J.; Faubion, M. G.; Gastfriend, B. D.; Palecek, S. P.; Shusta, E. V. An isogenic neurovascular unit model comprised of human induced pluripotent stem cellderived brain microvascular endothelial cells, pericytes, astrocytes, and neurons. Fluids Barriers CNS. 2019, 16, 25.

(19) Vatine, G. D.; Barrile, R.; Workman, M. J.; Sances, S.; Barriga, B. K.; Rahnama, M.; Barthakur, S.; Kasendra, M.; Lucchesi, C.; Kerns, J.; Wen, N.; Spivia, W. R.; Chen, Z.; Van Eyk, J.; Svendsen, C. N. Human iPSC-Derived Blood-Brain Barrier Chips Enable Disease Modeling and Personalized Medicine Applications. Cell Stem Cell. 2019, 24, 995-1005.

(20) Hodge, R. D.; Bakken, T. E.; Miller, J. A.; Smith, K. A.; Barkan, E. R.; Graybuck, L. T.; Close, J. L.; Long, B.; Johansen, N.; Penn, O.; Yao, Z.; Eggermont, J.; Höllt, T.; Levi, B. P.; Shehata, S. I.; Aevermann, B.; Beller, A.; Bertagnolli, D.; Brouner, K.; Casper, T.; Cobbs, C.; Dalley, R.; Dee, N.; Ding, S.-L.; Ellenbogen, R. G.; Fong, O.; Garren, E.; Goldy, J.; Gwinn, R. P.; Hirschstein, D.; Keene, C. D.; Keshk, M.; Ko, A. L.; Lathia, K.; Mahfouz, A.; Maltzer, Z.; McGraw, M.; Nguyen, T. N.; Nyhus, J.; Ojemann, J. G.; Oldre, A.; Parry, S.; Reynolds, S.; Rimorin, C.; Shapovalova, N. V.; Somasundaram, S.; Szafer, A.; Thomsen, E. R.; Tieu, M.; Quon, G.; Scheuermann, R. H.; Yuste, R.; Sunkin, S. M.; Lelieveldt, B.; Feng, D.; Ng, L.; Bernard, A.; Hawrylycz, M.; Phillips, J. W.; Tasic, B.; Zeng, H.; Jones, A. R.; Koch, C.; Lein, E. S. Conserved cell types with divergent features in human versus mouse cortex. Nature. 2019, 573, 61-68. 
(21) Takahashi, K.; Tanabe, K.; Ohnuki, M.; Narita, M.; Ichisaka, T.; Tomoda, K.; Yamanaka, S., Induction of pluripotent stem cells from adult human fibroblasts by defined factors. Cell. 2007, $131,861-872$.

(22) Lancaster, M. A.; Renner, M.; Martin, C. A.; Wenzel, D.; Bicknell, L. S.; Hurles, M. E.; Homfray, T.; Penninger, J. M.; Jackson, A. P.; Knoblich, J. A. Cerebral organoids model human brain development and microcephaly. Nature. 2013, 501, 373-379.

(23) Chiaradia, I.; Lancaster, M. A. Brain organoids for the study of human neurobiology at the interface of in vitro and in vivo. Nat. Neurosci. 2020, 23, 1496-1508.

(24) Wimmer, R. A.; Leopoldi, A.; Aichinger, M.; Wick, N.; Hantusch, B.; Novatchkova, M.; Taubenschmid, J.; Hammerle, M.; Esk, C.; Bagley, J. A.; Lindenhofer, D.; Chen, G.; Boehm, M.; Agu, C. A.; Yang, F.; Fu, B.; Zuber, J.; Knoblich, J. A.; Kerjaschki, D.; Penninger, J. M. Human blood vessel organoids as a model of diabetic vasculopathy. Nature. 2019, 565, 505-510.

(25) Chen, A.; Guo, Z.; Fang, L.; Bian, S. Application of Fused Organoid Models to Study Human Brain Development and Neural Disorders. Front. Cell. Neurosci. 2020, 14, 133.

(26) Sakaguchi, H.; Ozaki, Y.; Ashida, T.; Matsubara, T.; Oishi, N.; Kihara, S.; Takahashi, J. SelfOrganized Synchronous Calcium Transients in a Cultured Human Neural Network Derived from Cerebral Organoids. Stem Cell Rep. 2019, 13, 458-473.

(27) Yamamizu, K.; Iwasaki, M.; Takakubo, H.; Sakamoto, T.; Ikuno, T.; Miyoshi, M.; Kondo, T.; Nakao, Y.; Nakagawa, M.; Inoue, H.; Yamashita, J. K. In Vitro Modeling of Blood-Brain Barrier with Human iPSC-Derived Endothelial Cells, Pericytes, Neurons, and Astrocytes via Notch Signaling. Stem Cell Rep. 2017, 8, 634-647. 
(28) Ribecco-Lutkiewicz, M.; Sodja, C.; Haukenfrers, J.; Haqqani, A. S.; Ly, D.; Zachar, P.; Baumann, E.; Ball, M.; Huang, J.; Rukhlova, M.; Martina, M.; Liu, Q.; Stanimirovic, D.; Jezierski, A.; Bani-Yaghoub, M. A novel human induced pluripotent stem cell blood-brain barrier model: Applicability to study antibody-triggered receptor-mediated transcytosis. Sci Rep. 2018, 8, 1873.

(29) Gunhanlar, N.; Shpak, G.; van der Kroeg, M.; Gouty Colomer, L. A.; Munshi, S. T.; Lendemeijer, B.; Ghazvini, M.; Dupont, C.; Hoogendijk, W. J. G.; Gribnau, J.; de Vrij, F. M. S.; Kushner, S. A. A simplified protocol for differentiation of electrophysiologically mature neuronal networks from human induced pluripotent stem cells. Mol. Psychiatry. 2018, 23, 1336-1344.

(20) Teller, S.; Granell, C.; De Domenico, M.; Soriano, J.; Gomez, S.; Arenas, A. Emergence of assortative mixing between clusters of cultured neurons. PLoS Comput Biol. 2014, 10, e1003796.

(31) Jeong, G. S.; Chang, J. Y.; Park, J. S.; Lee, S. A.; Park, D.; Woo, J.; An, H.; Lee, C. J.; Lee, S. H. Networked neural spheroid by neuro-bundle mimicking nervous system created by topology effect. Mol. Brain. 2015, 8, 17.

(32) Girão, A. F.; Sousa, J.; Domínguez Bajo, A.; González Mayorga, A.; Bdikin, I.; Pujades Otero, E.; Casañ Pastor, N.; Hortigüela, M. J.; Otero Irurueta, G.; Completo, A.; Serrano, M. C.; Marques, P. A. A. P. 3D Reduced Graphene Oxide Scaffolds with a Combinatorial Fibrous-Porous Architecture for Neural Tissue Engineering. ACS Appl. Mater. Interfaces. 2020, 12, 38962-38975.

(33) Dingle, Y. T. L.; Liaudanskaya, V.; Finnegan, L. T.; Berlind, K. C.; Mizzoni, C.; Georgakoudi, I.; Nieland, T. J. F.; Kaplan, D. L. Functional Characterization of ThreeDimensional Cortical Cultures for In Vitro Modeling of Brain Networks. iScience. 2020, 23, 101434. 
(34) Pagan Diaz, G. J.; Ramos Cruz, K. P.; Sam, R.; Kandel, M. E.; Aydin, O.; Saif, M. T. A.; Popescu, G.; Bashir, R. Engineering geometrical 3-dimensional untethered in vitro neural tissue mimic. PNAS. 2019, 116, 25932.

(35) Chalard, A.; Vaysse, L.; Joseph, P.; Malaquin, L.; Souleille, S.; Lonetti, B.; Sol, J. C.; Loubinoux, I.; Fitremann, J. Simple Synthetic Molecular Hydrogels from Self-Assembling Alkylgalactonamides as Scaffold for 3D Neuronal Cell Growth. ACS Appl. Mater. Interface. 2018, 10, 17004-17017.

(36) Pautot, S.; Wyart, C.; Isacoff, E. Y. Colloid-guided assembly of oriented 3D neuronal networks. Nat. Methods. 2008, 5, 735-740.

(37) Liu, Y. C.; Lee, I. C.; Lei, K. F. Toward the Development of an Artificial Brain on a Micropatterned and Material-Regulated Biochip by Guiding and Promoting the Differentiation and Neurite Outgrowth of Neural Stem/Progenitor Cells. ACS Appl. Mater. Interface. 2018, 10, 52695277.

(38) Park, J.; Wetzel, I.; Marriott, I.; Dréau, D.; D’Avanzo, C.; Kim, D. Y.; Tanzi, R. E.; Cho, H. A 3D human triculture system modeling neurodegeneration and neuroinflammation in Alzheimer's disease. Nat. Neurosci. 2018, 21, 941-951.

(39) Holloway, P. M.; Willaime Morawek, S.; Siow, R.; Barber, M.; Owens, R. M.; Sharma, A. D.; Rowan, W.; Hill, E.; Zagnoni, M. Advances in microfluidic in vitro systems for neurological disease modeling. J. Neurosci. Res. 2021, 99, 1276-1307.

(40) Stone, N. L.; England, T. J.; O’Sullivan, S. E. A Novel Transwell Blood Brain Barrier Model Using Primary Human Cells. Front. Cell. Neurosci. 2019, 13, 230. 
(41) Reed, M. J.; Damodarasamy, M.; Banks, W. A. The extracellular matrix of the blood-brain barrier: structural and functional roles in health, aging, and Alzheimer's disease. Tissue Barriers. 2019, 7, 1651157.

(42) Tang, Y.; Liu, L.; Li, J.; Yu, L.; Wang, L.; Shi, J.; Chen, Y. Induction and differentiation of human induced pluripotent stem cells into functional cardiomyocytes on a compartmented monolayer of gelatin nanofibers. Nanoscale. 2016, 8, 14530-14540.

(43) Tang, Y.; Liu, L.; Li, J.; Yu, L.; Severino, F. P. U.; Wang, L.; Shi, J.; Tu, X.; Torre, V.; Chen, Y. Effective motor neuron differentiation of hiPSCs on a patch made of crosslinked monolayer gelatin nanofibers. J. Mater. Chem. B. 2016, 4, 3305-3312.

(44) Wang, B.; Wang, L.; Tang, Y.; Shi, J.; Wei, J.; Tu, X.; Chen, Y. Fabrication of spaced monolayers of electrospun nanofibers for three-dimensional cell infiltration and proliferation. Microelectro. Eng. 2018, 198, 73-77.

(45) Patel, T. P.; Man, K.; Firestein, B. L.; Meaney, D. F., Automated quantification of neuronal networks and single-cell calcium dynamics using calcium imaging. J Neurosci Methods. 2015, $243,26-38$.

(46) Rofaani, E.; Peng, J.; Wang, L.; He, Y.; Huang, B.; Chen, Y. Fabrication of ultrathin artificial basement membrane for epithelial cell culture. Microelectro. Eng. 2020, 232, 111407.

(47) Mederos, S.; González Arias, C.; Perea, G. Astrocyte-Neuron Networks: A Multilane Highway of Signaling for Homeostatic Brain Function. Front. Synaptic Neurosci. 2018, 10, 45.

(48) Durkee, C. A.; Araque, A. Diversity and Specificity of Astrocyte-neuron Communication. Neuroscience. 2019, 396, 73-78. 
(49) Gong, L.; Cao, L.; Shen, Z.; Shao, L.; Gao, S.; Zhang, C.; Lu, J.; Li, W. Materials for Neural Differentiation, Trans-Differentiation, and Modeling of Neurological Disease. Advanced Materials. 2018, 30, 1705684.

(50) Kang, S. M.; Kim, D.; Lee, J. H.; Takayama, S.; Park, J. Y. Engineered Microsystems for Spheroid and Organoid Studies. Adv. Healthc. Mater. 2021, 10, 2001284.

(51) Geraili, A.; Jafari, P.; Hassani, M. S.; Araghi, B. H.; Mohammadi, M. H.; Ghafari, A. M.; Tamrin, S. H.; Modarres, H. P.; Kolahchi, A. R.; Ahadian, S.; Sanati Nezhad, A. Controlling Differentiation of Stem Cells for Developing Personalized Organ-on-Chip Platforms. Adv. Healthc. Mater 2018, 7, 1700426.

(52) Tang, Y.; Ulloa Severino, F. P.; Iseppon, F.; Torre, V.; Chen, Y. Patch method for culture of primary hippocampal neurons. Microelectro. Eng. 2017, 175, 61-66.

(53) Xie, J.; MacEwan, M. R.; Schwartz, A. G.; Xia, Y., Electrospun nanofibers for neural tissue engineering. Nanoscale. 2010, 2, 35-44.

(54) Liu, L.; Yoshioka, M.; Nakajima, M.; Ogasawara, A.; Liu, J.; Hasegawa, K.; Li, S.; Zou, J.; Nakatsuji, N.; Kamei, K.; Chen, Y. Nanofibrous gelatin substrates for long-term expansion of human pluripotent stem cells. Biomaterials. 2014, 35, 6259-6267.

(55) Jakobsson, A.; Ottosson, M.; Zalis, M. C.; O'Carroll, D.; Johansson, U. E.; Johansson, F. Three-dimensional functional human neuronal networks in uncompressed low-density electrospun fiber scaffolds. Nanomedicine. 2017, 13, 1563-1573.

(56) Huang, Z.; Jiang, X. Micro/nano-scale materials and structures for constructing neuronal networks and addressing neurons. J. Mater. Chem. C. 2013, 1, 7652-7662. 
(57) Kim, J. I.; Hwang, T. I.; Aguilar, L. E.; Park, C. H.; Kim, C. S. A Controlled Design of Aligned and Random Nanofibers for 3D Bi-functionalized Nerve Conduits Fabricated via a Novel Electrospinning Set-up. Sci Rep. 2016, 6, 23761.

(58) Rose, J. C.; De Laporte, L. Hierarchical Design of Tissue Regenerative Constructs. Adv. Healthc. Mater. 2018, 7, e1701067.

(59) Gibson, L. J.; Ashby, M. F. Cellular Solids: Structure and Properties. 2 ed. Cambridge University Press: Cambridge, 1997, 93-174.

(60) Kuiper, S.; Brink, R.; Nijdam, W.; Krijnen, G. J. M.; Elwenspoek, M. C. Ceramic microsieves: influence of perforation shape and distribution on flow resistance and membrane strength. J. Membr. Sci. 2002, 196, 149-157.

(61) Obenaus, A. M.; Mollica, M. Y.; Sniadecki, N. J. (De)form and Function: Measuring Cellular Forces with Deformable Materials and Deformable Structures. Adv. Healthc. Mater. 2020, 9, e1901454.

(62) Li, J.; Zhang, F.; Yu, L.; Fujimoto, N.; Yoshioka, M.; Li, X.; Shi, J.; Kotera, H.; Liu, L.; Chen, Y. Culture substrates made of elastomeric micro-tripod arrays for long-term expansion of human pluripotent stem cells. J. Mater. Chem. B. 2017, 5, 236-244.

(63) Oddo, A.; Peng, B.; Tong, Z.; Wei, Y.; Tong, W. Y.; Thissen, H.; Voelcker, N. H. Advances in Microfluidic Blood-Brain Barrier (BBB) Models. Trends Biotechnol. 2019, 37, 1295-1314.

(64) Shetty, A. K.; Zanirati, G. The Interstitial System of the Brain in Health and Disease. Aging Dis. 2020, 11, 200-211. 
(65) Pitingolo, G.; He, Y.; Huang, B.; Wang, L.; Shi, J.; Chen, Y. An automatic cell culture platform for differentiation of human induced pluripotent stem cells. Microelectro Eng. 2020, 231, 111371.

GRAPHYSICAL ABSTRACT

Multiscale scaffolds

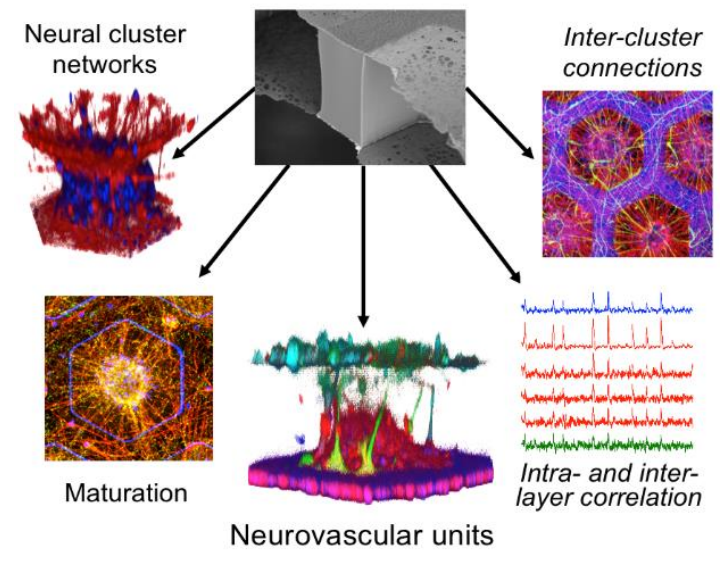

